\title{
A SIR model on a refining spatial grid - Law of Large Numbers
}

\author{
M. N'zi * \\ E. Pardoux ${ }^{\dagger}$ \\ T. Yeo
}

July 23, 2020

\begin{abstract}
We study in this paper a compartmental SIR model for a population distributed in a bounded domain $\mathrm{D}$ of $\mathbb{R}^{d}, \mathrm{~d}=1,2$ or 3 . We describe a spatial model for the spread of a disease on a grid of $\mathrm{D}$. We prove two laws of large numbers. On the one hand, we prove that the stochastic model converges to the corresponding deterministic patch model as the size of the population tends to infinity. On the other hand, by letting both the size of the population tend to infinity and the mesh of the grid go to zero, we obtain a law of large numbers in the supremum norm, where the limit is a diffusion SIR model in D.
\end{abstract}

Keywords: spatial model, deterministic, stochastic, law of large numbers

Funding: Ténan Yeo was supported by a thesis scholarship from the government of Ivory Coast, and a salary as instructor at University of Aix-Marseille, and the two other authors by their respective university.

Conflict of Interest: The authors declare that they have no conflict of interest.

\section{Introduction}

There is by now a good number of books and a huge number of papers treating mathematical models of epidemics. Most of them treat deterministic models, while some of them discuss as well stochastic models. Let us quote among many others Kermack \& McKendrick (1927), Anderson \& Britton (2000), Britton \& Pardoux (2019). These last two works show that the standard deterministic models are law of large numbers limits of individual-based stochastic models. They also study fluctuations around the law of large numbers limit, via the central limit theorem, and concerning the last reference, the

\footnotetext{
*Univ Félix Houphouët Boigny, modestenzi@yahoo.fr

${ }^{\dagger}$ Aix Marseille Univ, CNRS, Centrale Marseille, I2M, Marseille, France, etienne.pardoux@univ-amu.fr

‡Aix Marseille Univ, CNRS, Centrale Marseille, I2M, Marseille, France, tenan.yeo@univ-amu.fr
} 
large deviations. Those fluctuations allow to explain extinction of an endemic disease, which is a stable equilibrium of the deterministic model.

The classical SIR model ignores the fact that a population spreads over a spatial region. However environmental heterogeneity, spatial connectivity and movement of individuals play important roles in the spread of infectious diseases. Spatially uniform models are not sufficient to give a realistic picture of the spread of the disease. There is by now quite an important literature on spatial epidemics model, both in discrete and in continuous space, see e.g. Allen, Bolker, Lou \& Nevai (2007) and Allen, Bolker, Lou \& Nevai (2008), and the references therein.

In the present paper, we consider both deterministic and stochastic models in discrete and continuous space. More precisely, we start with an individual based stochastic model for a population with constant size $\mathbf{N} \varepsilon^{-d}$, distributed on the nodes of a regular grid discretizing $[0,1]^{d}$, with $d=1,2$ or 3 (we shall concentrate mainly on the case $d=2$, which seems to us most relevant). Letting first $\mathbf{N} \rightarrow \infty$, while $\varepsilon$, the mesh size, is kept fixed, we shall obtain as law of large numbers limit a system of ODEs on the grid, which is a patch epidemics model. Letting then $\varepsilon \rightarrow 0$, we will show that the system of ODEs converges to a system of PDEs on $[0,1]^{d}$, which is a deterministic epidemic model in continuous space. It is rather clear that one cannot hope to get the same result by letting first $\varepsilon \rightarrow 0$, and then $\mathbf{N} \rightarrow \infty$. Indeed, the first limit should be a continuous space model for quantities which take their values in the set $\{k / \mathbf{N}, 0 \leq k \leq \mathbf{N}\}$, with a partial differential operator for the displacement of the population, which would not make much sense. Consequently, if one wants to obtain a limit while letting jointly $\mathbf{N} \rightarrow \infty$ and $\varepsilon \rightarrow 0$, there must be a constraint which limits the speed of convergence of $\varepsilon$ to 0 , in terms of the speed of convergence of $\mathbf{N}$ to $+\infty$. The weakest possible such constraint seems to be the one which has been first introduced by Blount (1992) for chemical reaction models, namely the restriction that $\mathbf{N} / \log (1 / \varepsilon) \rightarrow \infty$, see also Debussche \& Nankep (2017). We shall extend that result to our situation where the limit is not a single PDE, but a system of PDEs.

The model is constructed on a $d$-dimensional bounded domain $[0,1]^{d}(d=1,2,3)$. We first suppose that the population is spatially distributed on the nodes of a grid $D_{\varepsilon}:=[0,1]^{d} \cap \varepsilon \mathbb{Z}^{d}=\left\{x_{i}, 1 \leq i \leq \varepsilon^{-d}\right\}$ of $[0,1]^{d}$, where $0<\varepsilon<1$ (two neighboring sites are at distance $\varepsilon$ apart, see Figure 1). Nodes represent communities in which the disease can grow. The population is divided in three compartments S, I and R. For a space-time coordinate $\left(\mathrm{t}, x_{i}\right)$, we denote by

- $S^{\varepsilon}\left(t, x_{i}\right)$ the number of susceptibles at site $x_{i}$ at time $t$,

- $I^{\varepsilon}\left(t, x_{i}\right)$ the number of infected at site $x_{i}$ at time $t$,

- $R^{\varepsilon}\left(t, x_{i}\right)$ the number of removed at site $x_{i}$ at time $t$.

In this case the deterministic model is given by a system of ordinary differential equation (ODE) and the stochastic one by a jump Markov process. Note that Arnold \& Theodosopulu (1980), Kotelenez (1986), Blount (1992), and also some of the references therein, describe such spatial models for chemical reactions. The resulting process has one component and is compared with the corresponding deterministic model. 
In the present paper, we focus our attention on the law of large numbers. In future works, we intend to discuss the fluctuations around the law of large numbers.

Let us briefly describe the content of this paper. In section 1, we introduce a deterministic model on the grid $D_{\varepsilon}$ of the bounded domain $[0,1]^{d}$ and we recall the relation between this model and the limiting PDE model on $[0,1]^{d}$ as $\varepsilon \rightarrow 0$. Then we introduce the stochastic model on the same grid for a population of total size $\mathbf{N} \varepsilon^{-d}$. In section 2, we fix the parameter $\varepsilon$ and let the initial average number $\mathbf{N}$ of individuals in each site tend to infinity: the limiting law of large numbers limit is the already introduced deterministic model. As $\varepsilon \rightarrow 0$ our system of ODEs converges towards a system of PDEs. Finally in section 3, we prove a law of large numbers in the supremum norm when we let both the size of the population go to infinity and the mesh of the grid go to zero, under the weak restriction that $\frac{\mathbf{N}}{\log (1 / \mathcal{E})} \longrightarrow \infty$.

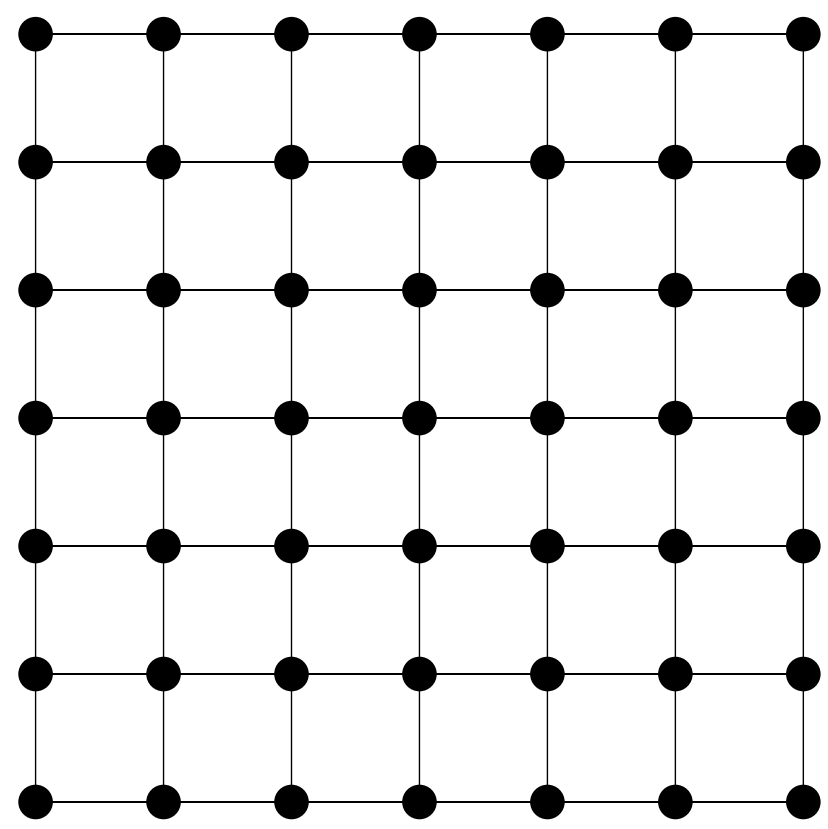

Figure 1- $[0,1] \times[0,1]$ grid

\section{The models}

Suppose that individuals are living in the bounded domain $D:=(0,1)^{d} \subset \mathbb{R}^{d}$. We consider an infectious disease which spreads in the population. Consider at each point of a grid (see Figure 1 ) on the ddimensional domain $D$ a deterministic and a stochastic SIR model, with migration between neighboring sites (two neighboring sites are at distance $\varepsilon$ apart). We assume that the mesh size of the grid $\varepsilon$ is such that $\varepsilon^{-1} \in \mathbb{N}$, where $\mathbb{N}$ is the set of positive integers. We assume that the studied epidemic concerns a population of fixed size. In this model, infections are local. We let $\beta: \mathbb{R}^{d} \longrightarrow \mathbb{R}_{+}$and $\alpha: \mathbb{R}^{d} \longrightarrow \mathbb{R}_{+}$ be continuous functions and we set $\bar{\beta}=\sup _{x \in D} \beta(x)$ and $\bar{\alpha}=\sup _{x \in D} \alpha(x)$. For each site $x_{i}$

- Susceptible individuals become infectious at rate $\beta\left(\underset{x \in D}{\left(x_{i}\right)} \frac{S^{\varepsilon}\left(t, x_{i}\right)}{S^{\varepsilon}\left(t, x_{i}\right)+I^{\varepsilon}\left(t, x_{i}\right)+R^{\varepsilon}\left(t, x_{i}\right)} I^{\varepsilon}\left(t, x_{i}\right)\right.$.

Note that an individual chosen uniformly at random site $x_{i}$ at time $t$ is susceptible with probability 


$$
\frac{S^{\varepsilon}\left(t, x_{i}\right)}{S^{\varepsilon}\left(t, x_{i}\right)+I^{\varepsilon}\left(t, x_{i}\right)+R^{\varepsilon}\left(t, x_{i}\right)}
$$

- each infectious recovers at rate $\alpha\left(x_{i}\right)$, so the total recovery rate at time $\mathrm{t}$ is $\alpha\left(x_{i}\right) I^{\varepsilon}\left(t, x_{i}\right)$;

- the migrations of susceptible, infected and removed individuals between location $x_{i}$ and its neighboring sites occur at rate $\frac{\mu_{S}}{\varepsilon^{2}} S^{\varepsilon}\left(t, x_{i}\right), \frac{\mu_{I}}{\varepsilon^{2}} I^{\varepsilon}\left(t, x_{i}\right)$ and $\frac{\mu_{R}}{\varepsilon^{2}} R^{\varepsilon}\left(t, x_{i}\right)$ respectively. $\mu_{S}, \mu_{I}$ and $\mu_{R}$ are positive diffusion coefficients for the susceptible, infected and removed subpopulations, respectively.

Here, we assume that the compartment $R$ contains individuals who are dead or who have recovered and have permanent immunity. We can assume boundary conditions of the Neumann or periodic type. In this paper, we focus our attention on Neumann boundary conditions (representing a closed environment i.e. there is no flux of individuals through the boundary). The choice $D=(0,1)^{d}$ as the spatial domain is made for the sake of simplifying the analysis, but our results can be extended to any bounded domain $D \subset \mathbb{R}^{d}$, with a reasonably smooth boundary.

Initially $\mathbf{N} \varepsilon^{-d}$ individuals are distributed on the grid. That is, there is an average of $\mathbf{N}$ individuals on each site. We first introduce the deterministic model and then we construct the corresponding stochastic model.

In the following we use the generic notation $C$ for a positive constant, the value of which may change from line to line. These constants can depend upon some parameters of the model, as long as these are independent of $\varepsilon$ and $\mathbf{N}$.

\subsection{The deterministic model}

The space is the grid $D_{\varepsilon}$ of D. In order to take into account Neumann boundary conditions, we add some fictitious sites which extend the grid outside the domain, as shown in Figure 2 below. We denote by $\partial_{\vec{n} \text {.out }} D_{\varepsilon}$ the set of those fictitious sites. We use the notation $y_{i} \sim x_{i}$ to mean that the sites $y_{i}$ and $x_{i}$ are neighbors. Each interior point of $D_{\varepsilon}$ has $2 d$ neighbours. Each boundary point has at least one fictitious site among its neighbors. 


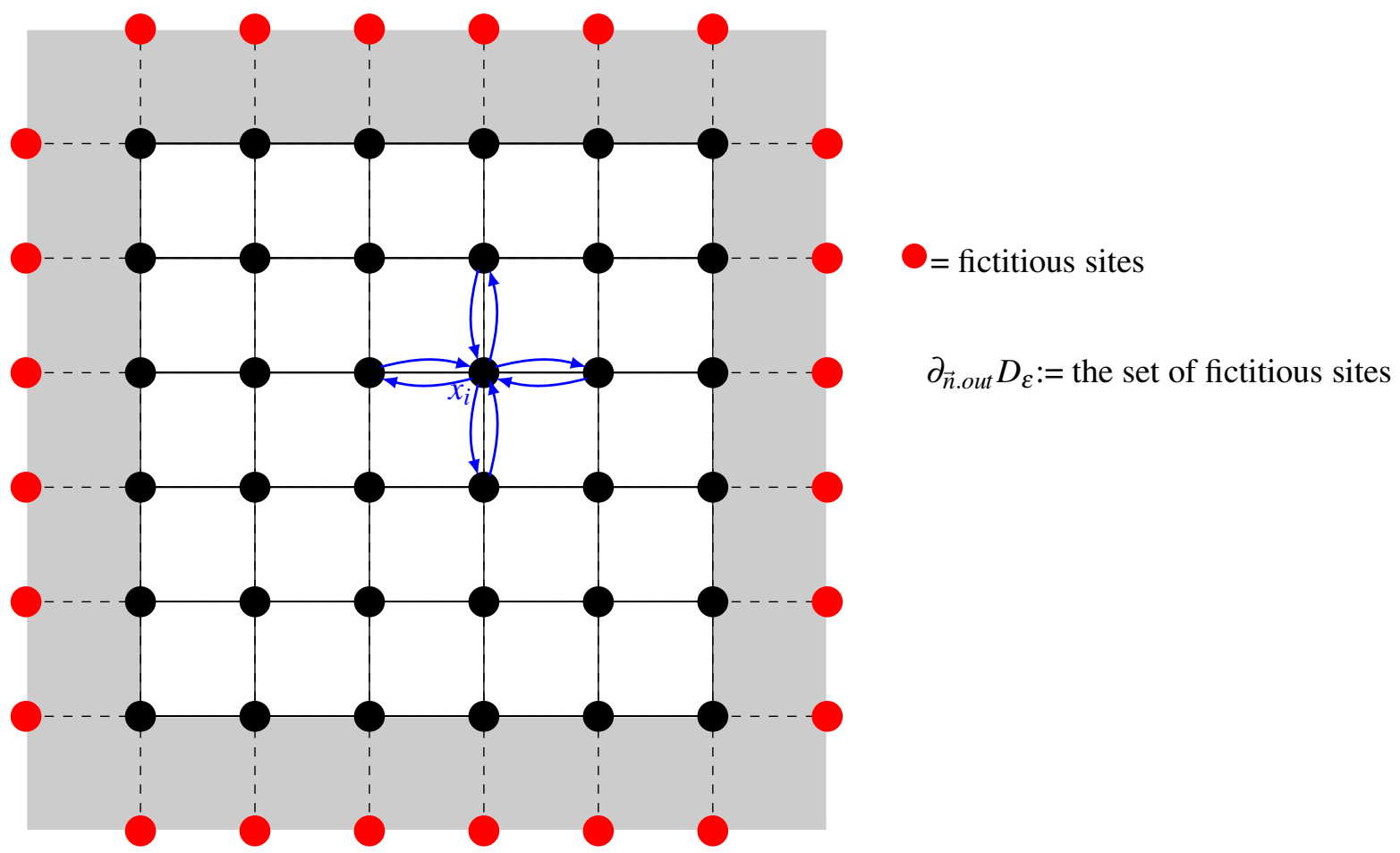

Figure 2-Modeling the Neumann condition

By thinking of an infinite size population allowing "proportions" in each compartment to be continuous, we have the following deterministic model for "proportions" (this point of view will become quite clear in section 2 below):

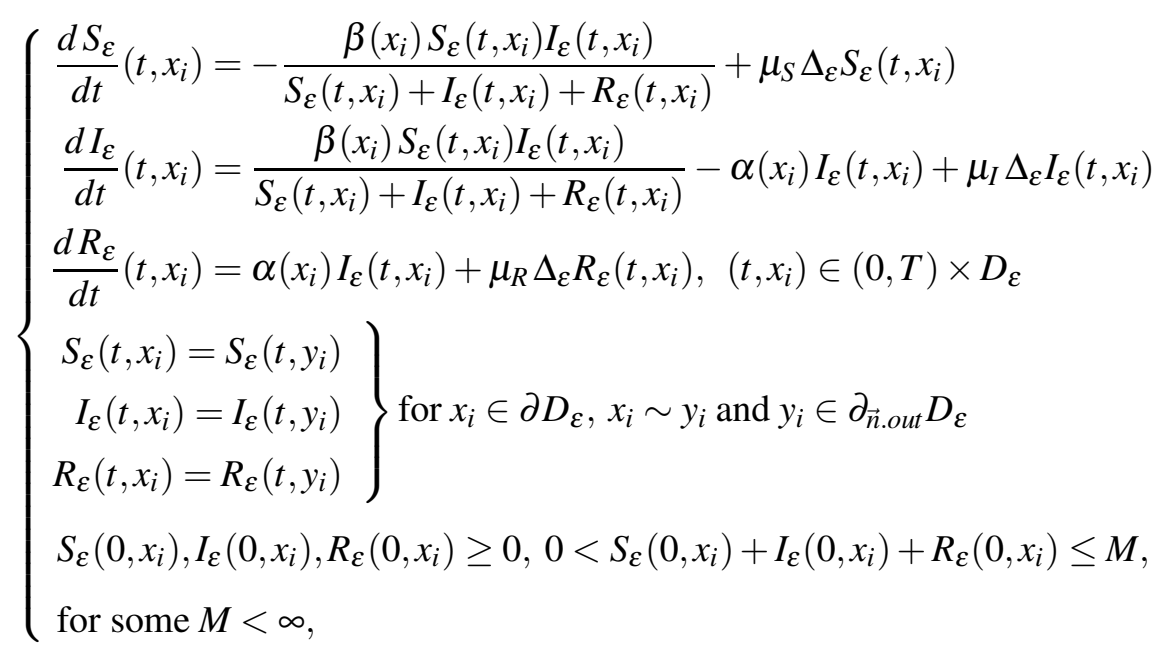

where $S_{\varepsilon}\left(t, x_{i}\right)$ (resp. $I_{\varepsilon}\left(t, x_{i}\right)$, resp. $\left.R_{\varepsilon}\left(t, x_{i}\right)\right)$ is the proportion of the total population which is both susceptible (resp. infectious, resp. removed) and located at site $x_{i}$ at time $t . \Delta_{\varepsilon}$ is the discrete Laplace operator defined as follows: $\Delta_{\varepsilon} f\left(x_{i}\right)=\varepsilon^{-2} \sum_{j=1}^{d}\left[f\left(x_{i}+\varepsilon e_{j}\right)-2 f\left(x_{i}\right)+f\left(x_{i}-\varepsilon e_{j}\right)\right]$. 
Note that (1.1) is the discrete space approximation of the following system of PDE

$$
(1.2)\left\{\begin{array}{l}
\frac{\partial \mathbf{s}}{\partial t}(t, x)=-\frac{\beta(x) \mathbf{s}(t, x) \mathbf{i}(t, x)}{\mathbf{s}(t, x)+\mathbf{i}(t, x)+\mathbf{r}(t, x)}+\mu_{S} \Delta \mathbf{s}(t, x) \\
\frac{\partial \mathbf{i}}{\partial t}(t, x)=\frac{\beta(x) \mathbf{s}(t, x) \mathbf{i}(t, x)}{\mathbf{s}(t, x)+\mathbf{i}(t, x)+\mathbf{r}(t, x)}-\alpha(x) \mathbf{i}(t, x)+\mu_{I} \Delta \mathbf{i}(t, x) \\
\frac{\partial \mathbf{r}}{\partial t}(t, x)=\alpha(x) \mathbf{i}(t, x)+\mu_{S} \Delta \mathbf{r}(t, x), \quad(t, x) \in(0, T) \times D \\
\frac{\partial \mathbf{s}}{\partial n_{\text {out }}}(t, x)=\frac{\partial \mathbf{i}}{\partial n_{\text {out }}}(t, x)=\frac{\partial \mathbf{r}}{\partial n_{\text {out }}}(t, x)=0, \quad \text { for } x \in \partial D \\
\mathbf{s}(0, x), \mathbf{i}(0, x), \mathbf{r}(0, x) \geq 0,0<\mathbf{s}(0, x)+\mathbf{i}(0, x)+\mathbf{r}(0, x) \leq M
\end{array}\right.
$$

where $\frac{\partial}{\partial n_{\text {out }}}$ denotes differentiation in the direction of the outward normal to $\partial D$ and $\Delta$ denotes the ddimensional Laplace operator.

System (1.2) is a reaction-diffusion epidemic model which has been studied by several authors. Webb (1981) gave a similar reaction-diffusion model for a deterministic diffusive epidemic model, established the existence of solutions and analyzed their behavior as $t \rightarrow \infty$. His method exploits tools of functional analysis and dynamical systems, specifically the theory of semigroups of linear and nonlinear operators in Banach spaces and Lyapunov stability techniques for dynamical systems in metric spaces. In the same way Yamazaki \& Wang (2016) gave a reaction-convection-diffusion epidemic model for cholera dynamics and studied the global well-posedness and the asymptotic behavior of the solutions. See also Du \& Peng (2016), Yamazaki (2018a), Yamazaki (2018b). Let us mention that the SIR model (1.1) describes the spread of an infectious disease where recovered individuals gain immunity from re-infection. Of course in some cases recovered individuals have not permanent immunity. Hence individuals in the compartiment $\mathrm{R}$ can experience reinfection. Moreover, susceptible individuals that become infected can first pass through a latent stage (exposed). Such models are used to study the transmission dynamics of the Ebola virus disease as treated in Agusto (2017a). Also, in Agusto et al. (2017b) the authors used such model to explore the Zika virus transmission dynamics in a human population. Another model which received attention in the literature is the diffusion epidemic SIS model. In this model, when an infectious individual cures, he immediately becomes susceptible again. Such model has been considered in Allen et al. (2008). Although we restrict ourselves to the SIR model, our results can easily be adapted to SIRS, SIS, SEIR, SEIRS models.

Before describing the stochastic model, we introduce some notations and preliminaries, and then discuss the relation between the system of PDEs (1.2) and its discretisation.

\subsubsection{Some notations and preliminaries}

In this subsection we introduce some notations and also give preliminary lemmas which will be needed in our subsequent work. For all $x_{i} \in D_{\varepsilon}$, let $V_{i}$ be the cube centered at the site $x_{i}$ with volume $\varepsilon^{d}$. Let 
$H^{\varepsilon} \subset L^{2}(D)$ denote the space of real valued step functions that are constant on each cell $V_{i}$. For $f \in H^{\varepsilon}$, let us define

$$
\begin{aligned}
& \nabla_{\varepsilon}^{j,+} f\left(x_{i}\right)=\frac{f\left(x_{i}+\varepsilon e_{j}\right)-f\left(x_{i}\right)}{\varepsilon}, \\
& \nabla_{\varepsilon}^{j,-} f\left(x_{i}\right)=\frac{f\left(x_{i}\right)-f\left(x_{i}-\varepsilon e_{j}\right)}{\varepsilon} .
\end{aligned}
$$

It is not hard to see that

$$
\begin{aligned}
& \left\langle\nabla_{\varepsilon}^{j,+} f, g\right\rangle=-\left\langle f, \nabla_{\varepsilon}^{j,-} g\right\rangle, \\
& \Delta_{\varepsilon} f\left(x_{i}\right)=\sum_{j=1}^{d} \nabla_{\varepsilon}^{j,-} \nabla_{\varepsilon}^{j,+} f\left(x_{i}\right) .
\end{aligned}
$$

We introduce the canonical projection $P_{\varepsilon}: L^{2}(D) \longrightarrow H^{\varepsilon}$ given by

$$
\varphi \longmapsto P_{\varepsilon} \varphi(x)=\varepsilon^{-d} \int_{V_{i}} \varphi(y) d y \quad \text { if } x \in V_{i}
$$

Throughout this paper, we assume that the initial condition satisfies

Assumption $1.1 \mathcal{S}_{\varepsilon}(0, x)=P_{\varepsilon} \mathbf{s}(0, x), \mathcal{I}_{\varepsilon}(0, x)=P_{\varepsilon} \mathbf{i}(0, x), \mathcal{R}_{\varepsilon}(0, x)=P_{\varepsilon} \mathbf{r}(0, x)$ and

$$
\int_{D}(\mathbf{s}(0, x)+\mathbf{i}(0, x)+\mathbf{r}(0, x)) d x=1 .
$$

Here, we describe some of the spectral properties of the (discrete)-Laplacian which will play an important role in the sequel. More details can be found in Kotelenez(1986).

- For a multiindex $m=\left(m_{1}, \ldots, m_{d}\right)$, where $m_{j} \in \mathbb{N} \cup\{0\}$, and $x \in \mathbb{R}$, we define

$$
f_{m_{j}}(x)=\left\{\begin{array}{cl}
\sqrt{2} \cos \left(m_{j} \pi x\right), & \text { for } m_{j} \geq 1 \\
1, & \text { for } m_{j}=0
\end{array}\right.
$$

For $\varphi, \psi \in L^{2}(D),\langle\varphi, \phi\rangle:=\int_{D} \varphi(r) \phi(r) d r$ denotes the scalar product in $L^{2}(D)$.

For each $m \in \mathbb{Z}_{+}^{d}, x=\left(x^{1}, \cdots, x^{d}\right) \in D$, we define $\mathbf{f}_{m}(x)=\prod_{j=1}^{d} f_{m_{j}}\left(x^{j}\right) .\left\{\mathbf{f}_{m}, m \in \mathbb{Z}_{+}^{d}\right\}$ is a complete orthonormal system (CONS) of eigenvectors of $\Delta$ in $L^{2}(D)$ with eigenvalues $-\lambda_{m}=-\pi^{2} \sum_{j=1}^{d} m_{j}^{2}$. Consequently, the semigroup $\mathrm{T}(t):=\exp (\Delta t)$ acting on $L^{2}(D)$ generated by $\Delta$ can be represented by

$$
\mathrm{T}(t) \varphi=\sum_{m} \exp \left(-\lambda_{m} t\right)\left\langle\varphi, \mathbf{f}_{m}\right\rangle \mathbf{f}_{m}, \varphi \in L^{2}(D) .
$$

- For $i=\left(i_{1}, \ldots, i_{d}\right) \in\left\{0,1, \ldots, \varepsilon^{-1}-1\right\}^{d}$, let $V_{i}=\prod_{j=1}^{d}\left[\left(i_{j}-\frac{1}{2}\right) \varepsilon,\left(i_{j}+\frac{1}{2}\right) \varepsilon\right) \subset[0,1]^{d}$ and for $m \in\left\{0,1, \ldots, \varepsilon^{-1}\right\}^{d}$, we define $\mathbf{f}_{m}^{\varepsilon}(x)=\prod_{j=1}^{d} f_{m_{j}}\left(i_{j} \varepsilon\right)$ if $x \in V_{i} .\left\{\mathbf{f}_{m}^{\varepsilon}, m \in \mathbb{Z}_{+}^{d}\right\}$ form an orthonormal basis of $H^{\varepsilon}$ as a subspace of $L^{2}\left([0,1]^{d}\right)$ and are eigenfunctions of $\Delta_{\varepsilon}$ with eigenvalues 
$-\lambda_{m}^{\varepsilon}=-2 \varepsilon^{-2} \sum_{j=1}^{d}\left(1-\cos \left(m_{j} \pi \varepsilon\right)\right)$. Note that $\lambda_{m}^{\varepsilon} \longrightarrow \lambda_{m}$ as $\varepsilon \rightarrow 0$.

- Basic calculations show that there exists a constant $c$, such that for each $m_{j}, \varepsilon^{-2}\left(1-\cos \left(\pi m_{j} \varepsilon\right)\right)>$ $c m_{j}^{2}$.

- $\Delta_{\varepsilon}$ generates a contraction semigroup $\mathrm{T}_{\varepsilon}(t):=\exp \left(\Delta_{\varepsilon} t\right)$ represented on $H^{\varepsilon}$ by

$$
\mathrm{T}_{\varepsilon}(t) \varphi=\sum_{m} \exp \left(-\lambda_{m}^{\varepsilon} t\right)\left\langle\varphi, \mathbf{f}_{m}^{\varepsilon}\right\rangle \mathbf{f}_{m}^{\varepsilon}
$$

where the summation is taken on the $\varepsilon^{-d}$ eigenvectors of $\Delta_{\varepsilon}$. Note that both $\Delta_{\varepsilon}$ and $\mathrm{T}_{\varepsilon}(t)$ are self-adjoint and that $\mathrm{T}_{\varepsilon}(t) \Delta_{\varepsilon} \varphi=\Delta_{\varepsilon} \mathrm{T}_{\varepsilon}(t) \varphi$. Note also, for any $J \in\{S, I, R\}$, the semigroup generated by $\mu_{J} \Delta$ is $\mathrm{T}\left(\mu_{J} t\right)$. In the sequel, we will use the notation $\mathrm{T}_{J}(t):=\mathrm{T}\left(\mu_{J} t\right)$ and similarly, in the discrete case, we will use the notation $\mathrm{T}_{\varepsilon, J}(t):=\mathrm{T}_{\varepsilon}\left(\mu_{J} t\right)$. Also, for any $J \in\{S, I, R\}$, we let $\lambda_{m, J}:=\mu_{J} \lambda_{m}$ and $\lambda_{m, J}^{\varepsilon}:=\mu_{J} \lambda_{m}^{\varepsilon}$.

- We use $\|\varphi\|_{\infty}:=\sup _{x \in D}|\varphi(x)|$ to denote the supremum norm of $\varphi$ in $D$, and we define $\left\|\left(\begin{array}{c}\varphi \\ \phi\end{array}\right)\right\|_{\infty}:=\|\varphi\|_{\infty}+\|\phi\|_{\infty}$.

- If $Z$ is a space-time function, we use the notation $Z(t)=Z(t,$.$) .$

- For $n \geq 1, C^{n}(D)$ denotes the space of real valued continuous functions on $D$ with continuous partial derivatives of all orders from 1 to $n$. We use the standard partial ordering of $\mathbb{R}^{d}$ and the classical notations:

$u \leq v$ if, for all $1 \leq i \leq d, u_{i} \leq v_{i}$.

\subsubsection{Existence and uniqueness}

Let us set $X_{\varepsilon}=\left(S_{\varepsilon}, I_{\varepsilon}, R_{\varepsilon}\right)^{T}$. We introduce the function $G:(x ; u, v, w) \longmapsto\left(\begin{array}{c}-\frac{\beta(x) u v}{u+v+w} \\ \frac{\beta(x) u v}{u+v+w}-\alpha(x) v \\ \alpha(x) v\end{array}\right)$. We use the notation $\widetilde{\Delta}_{\varepsilon} X_{\varepsilon}=\left(\mu_{S} \Delta_{\varepsilon} S_{\varepsilon}, \mu_{I} \Delta_{\varepsilon} I_{\varepsilon}, \mu_{R} \Delta_{\varepsilon} R_{\varepsilon}\right)^{T}$. Then the compact form of system (1.1) is

$$
\left\{\begin{array}{c}
\frac{d X_{\mathcal{\varepsilon}}}{d t}\left(t, x_{i}\right)=\widetilde{\Delta}_{\varepsilon} X_{\mathcal{\varepsilon}}\left(t, x_{i}\right)+G\left(x_{i} ; X_{\mathcal{\varepsilon}}\left(t, x_{i}\right)\right),\left(t, x_{i}\right) \in(0, T) \times D_{\varepsilon} \\
X_{\mathcal{\varepsilon}}\left(t, x_{i}\right)=X_{\mathcal{\varepsilon}}\left(t, y_{i}\right), \text { for } x_{i} \in \partial D_{\mathcal{\varepsilon}}, x_{i} \sim y_{i} \text { and } y_{i} \in \partial_{\vec{n} . o u t} D_{\varepsilon} \\
X\left(0, x_{i}\right) \geq 0 \text { and } 0<S_{\mathcal{\varepsilon}}\left(0, x_{i}\right)+I_{\mathcal{E}}\left(0, x_{i}\right)+R_{\mathcal{\varepsilon}}\left(0, x_{i}\right) \leq M, \forall x_{i} \in D_{\varepsilon}
\end{array}\right.
$$

We have

Lemma 1.1 For each $\varepsilon>0$ fixed, the system (1.4) has a unique non-negative solution $X_{\varepsilon} \in C^{1}\left(\mathbb{R}_{+} ; \mathbb{R}_{+}^{3 \varepsilon^{-d}}\right)$. Moreover $\sup _{0 \leq t \leq T}\left\|X_{\mathcal{\varepsilon}}(t)\right\|_{\infty} \leq C(\bar{\alpha}, \bar{\beta})$.

Proof : Let us define $g: \mathbb{R}^{3} \longrightarrow \mathbb{R}$ 


$$
(u, v, w) \longmapsto g(u, v, w)=\frac{u v}{u+v+w}
$$

We have $G(x ; u, v, w)=\left(\begin{array}{c}-\beta(x) g(u, v, w) \\ \beta(x) g(u, v, w)-\alpha(x) v \\ \alpha(x) v\end{array}\right)$. We introduce

$g^{+}(u, v, w)=\left\{\begin{array}{cl}\frac{u^{+} v^{+}}{u^{+}+v^{+}+w^{+}}, & \text {if } u^{+}+v^{+}+w^{+}>0 ; \\ 0, & \text { otherwise, }\end{array}\right.$ where we used the notation $u^{+}=\sup (u, 0)$. We set

$G^{+}(x ; u, v, w)=\left(\begin{array}{c}-\beta(x) g^{+}(u, v, w) \\ \beta(x) g^{+}(u, v, w)-\alpha(x) v \\ \alpha(x) v\end{array}\right)$. Let us consider the system of ODEs

$(1.5)\left\{\begin{array}{l}\frac{d X_{\varepsilon}}{d t}\left(t, x_{i}\right)=\widetilde{\Delta}_{\varepsilon} X_{\varepsilon}\left(t, x_{i}\right)+G^{+}\left(x_{i} ; X_{\varepsilon}\left(t, x_{i}\right)\right), \quad\left(t, x_{i}\right) \in(0, T) \times D_{\varepsilon} \\ X_{\mathcal{\varepsilon}}\left(t, x_{i}\right)=X_{\varepsilon}\left(t, y_{i}\right), \text { for } x_{i} \in \partial D_{\mathcal{\varepsilon}}, x_{i} \sim y_{i} \text { and } y_{i} \in \partial_{\vec{n} . o u t} D_{\varepsilon} \\ X\left(0, x_{i}\right) \geq 0 \text { and } 0<S_{\mathcal{\varepsilon}}\left(0, x_{i}\right)+I_{\mathcal{\varepsilon}}\left(0, x_{i}\right)+R_{\mathcal{\varepsilon}}\left(0, x_{i}\right) \leq M, \forall x_{i} \in D_{\varepsilon} .\end{array}\right.$

Since $G^{+}$is globally Lipschitz and $\widetilde{\Delta}_{\varepsilon}$ is linear, then by the Picard-Lindelöf theorem the Cauchy problem (1.5) has a unique solution $\tilde{X}_{\varepsilon} \in C^{1}\left(\mathbb{R}_{+} ; \mathbb{R}^{3 \varepsilon^{-d}}\right)$. Now let us show that $X_{\varepsilon}(t) \geq 0$ for all $t \geq 0$. Let us set $t_{1}=\inf \left\{t>0\right.$ : there exists an index i such that $\left.S_{\varepsilon}\left(t, x_{i}\right)<0\right\}$. If $t_{1}<\infty$ then there exists $i_{1}$ such that $S_{\varepsilon}\left(t_{1}, x_{i_{1}}\right)=0$ and for all $j \neq i_{1} S_{\varepsilon}\left(t_{1}, x_{j}\right) \geq 0$. So that $\frac{d S_{\varepsilon}}{d t}\left(t_{1}, x_{i_{1}}\right) \geq 0$. Thus, either there exists an index $j$ such $x_{j} \sim x_{i_{1}}$ and $S_{\varepsilon}\left(t_{1}, x_{j}\right)>0$ or else $S_{\varepsilon}\left(t_{1}, x_{j}\right)=0$ for all $x_{j} \sim x_{i_{1}}$.

(i) In the first case $\frac{d S_{\varepsilon}}{d t}\left(t_{1}, x_{i_{1}}\right)>0$, which contradicts the definition of $t_{1}$.

(ii) Let us set $\mathrm{I}_{1}=\left\{x_{i} \in D_{\varepsilon}: S_{\varepsilon}\left(t_{1}, x_{i}\right)>0\right\}$. If $\mathrm{I}_{1}=\varnothing$ then $S_{\mathcal{\varepsilon}}\left(t_{1}, x_{i}\right)=0$, for all $x_{i} \in D_{\varepsilon}$. The uniqueness of the solution entails that the null vector is solution for the equations satisfied by $S_{\varepsilon}$ on the time interval $\left[t_{1},+\infty\right)$. We now assume that $I_{1} \neq \varnothing$, and define

$$
\begin{gathered}
\mathscr{V}_{1}\left(\mathrm{I}_{1}\right)=\left\{x_{i} \in D_{\varepsilon}: x_{i} \notin \mathrm{I}_{1}, \exists x_{j} \in \mathrm{I}_{1} \text { such that } x_{i} \sim x_{j}\right\}, \\
\mathscr{V}_{2}\left(\mathrm{I}_{1}\right)=\left\{x_{i} \in D_{\varepsilon}: x_{i} \notin \mathscr{V}_{1}\left(\mathrm{I}_{1}\right) \cup \mathrm{I}_{1}, \exists x_{j} \in \mathrm{I}_{1} \text { such that } x_{i} \sim x_{j}\right\}, \\
\vdots \\
\mathscr{V}_{k}\left(\mathrm{I}_{1}\right)=\left\{x_{i} \in D_{\varepsilon}: x_{i} \notin \mathscr{V}_{k-1}\left(\mathrm{I}_{1}\right) \cup \cdots \cup \mathscr{V}_{1}\left(\mathrm{I}_{1}\right) \cup \mathrm{I}_{1}, \exists x_{j} \in \mathscr{V}_{k-1}\left(\mathrm{I}_{1}\right) \text { such that } x_{i} \sim x_{j}\right\}, k \geq 1 .
\end{gathered}
$$

First, note that there exists a positive integer $k$ such that $\mathscr{V}_{k}\left(\mathrm{I}_{1}\right)=\varnothing$, because there is a finite number of sites. Now, if $x_{i} \in \mathscr{V}_{1}\left(\mathrm{I}_{1}\right)$, then $S_{\mathcal{\varepsilon}}\left(t_{1}, x_{i}\right)=0$ and $\frac{d S_{\mathcal{\varepsilon}}}{d t}\left(t_{1}, x_{i}\right)>0$, which contradicts the definition of $t_{1}$. Else, assume that $x_{i} \in \mathscr{V}_{2}\left(\mathrm{I}_{1}\right)$. On the one hand, we have $S_{\varepsilon}\left(t_{1}, x_{i}\right)=0, \frac{d S_{\varepsilon}}{d t}\left(t_{1}, x_{i}\right)=0$. On the other hand, 


$$
\text { since } \begin{aligned}
\frac{d^{2} S_{\varepsilon}}{d t^{2}}\left(t_{1}, x_{i}\right) & =-\beta\left(x_{i}\right) \frac{I_{\mathcal{\varepsilon}}\left(t_{1}, x_{i}\right) \frac{d S_{\varepsilon}}{d t}\left(t_{1}, x_{i}\right)+S_{\varepsilon}\left(t_{1}, x_{i}\right) \frac{d I_{\varepsilon}}{d t}\left(t_{1}, x_{i}\right)}{S_{\varepsilon}\left(t_{1}, x_{i}\right)+I_{\mathcal{\varepsilon}}\left(t_{1}, x_{i}\right)+R_{\varepsilon}\left(t_{1}, x_{i}\right)} \\
& +\beta\left(x_{i}\right) \frac{S_{\varepsilon}\left(t_{1}, x_{i}\right) I_{\varepsilon}\left(t_{1}, x_{i}\right)\left(\frac{d S_{\varepsilon}}{d t}\left(t_{1}, x_{i}\right)+\frac{d I_{\varepsilon}}{d t}\left(t_{1}, x_{i}\right)+\frac{d R_{\varepsilon}}{d t}\left(t_{1}, x_{i}\right)\right)}{\left(S_{\mathcal{\varepsilon}}\left(t_{1}, x_{i}\right)+I_{\varepsilon}\left(t_{1}, x_{i}\right)+R_{\varepsilon}\left(t_{1}, x_{i}\right)\right)^{2}}+\mu_{S} \Delta_{\varepsilon} \frac{d S_{\varepsilon}}{d t}\left(t_{1}, x_{i}\right),
\end{aligned}
$$

then $\frac{d^{2} S_{\varepsilon}}{d t^{2}}\left(t_{1}, x_{i}\right)=\mu_{S} \Delta_{\varepsilon} \frac{d S_{\varepsilon}}{d t}\left(t_{1}, x_{i}\right)>0$, because $x_{i} \in \mathscr{V}_{2}\left(\mathrm{I}_{1}\right)$, and we obtain again a contradiction. If $x_{i} \in \mathscr{V}_{j}\left(\mathrm{I}_{1}\right)$, for $j \geq 2$, we iterate the above argument to obtain a contradiction. Then in all cases we obtain a contradiction. So that $t_{1}=\infty$. Thus $S_{\mathcal{\varepsilon}}\left(t, x_{i}\right) \geq 0$ for all $\left(t, x_{i}\right) \in[0, T] \in D_{\mathcal{\varepsilon}}$. Similar arguments hold for $I_{\varepsilon}$ and $R_{\varepsilon}$. It follows from the positivity of the solution and the fact that $G=G^{+}$on $\mathbb{R}_{+}^{3}$, that the system (1.4) has a unique global solution $X_{\varepsilon} \in C^{1}\left(\mathbb{R}_{+} ; \mathbb{R}_{+}^{3 \varepsilon^{-d}}\right)$. Furthermore, writing the solution of (1.4) in its mild semigroup form, and using successively the fact that $\widetilde{\mathrm{T}}_{\varepsilon}:=\left(\mathrm{T}_{\varepsilon, S}, \mathrm{~T}_{\varepsilon, I}, \mathrm{~T}_{\varepsilon, R}\right)^{T}$ is a contraction semigroup on $\left(H^{\varepsilon},\left(L^{\infty}(D)\right)^{3}\right)$, the fact that $X\left(0, x_{i}\right) \leq 1$ for all $x_{i} \in D_{\varepsilon}$ and applying Gronwall's Lemma, we easily obtain that $\sup _{0 \leq t \leq T}\|X(t)\|_{\infty} \leq C(\bar{\alpha}, \bar{\beta})$. This concludes the proof of the lemma.

Let us now define $\mathcal{S}_{\mathcal{\varepsilon}}(t, x)=\sum_{i=1}^{\varepsilon^{-d}} S_{\mathcal{\varepsilon}}\left(t, x_{i}\right) \mathbf{1}_{V_{i}}(x), \mathcal{I}_{\mathcal{\varepsilon}}(t, x)=\sum_{i=1}^{\varepsilon^{-d}} I_{\mathcal{E}}\left(t, x_{i}\right) \mathbf{1}_{V_{i}}(x), \mathcal{R}_{\varepsilon}(t, x)=\sum_{i=1}^{\varepsilon^{-d}} R_{\mathcal{\varepsilon}}\left(t, x_{i}\right) \mathbf{1}_{V_{i}}(x)$, $\beta_{\varepsilon}(t, x)=\sum_{i=1}^{\varepsilon^{-d}} \beta\left(t, x_{i}\right) \mathbf{1}_{V_{i}}(x), \quad \alpha_{\varepsilon}(t, x)=\sum_{i=1}^{\varepsilon^{-d}} \alpha\left(t, x_{i}\right) \mathbf{1}_{V_{i}}(x)$, and we set $\mathcal{X}_{\varepsilon}=\left(\mathcal{S}_{\varepsilon}, \mathcal{I}_{\varepsilon}, \mathcal{R}_{\varepsilon}\right)^{T}$.

Note that the previous lemma is true with $\mathcal{X}_{\varepsilon}$ in place of $X_{\varepsilon}$. Let us set $X=(\mathbf{s}, \mathbf{i}, \mathbf{r})^{T}$. Then the compact form of the model (1.2) is

(1.6) $\left\{\begin{array}{l}\frac{\partial X}{\partial t}(t, x)=\widetilde{\Delta} X(t, x)+G(x ; X(t, x)), \quad(t, x) \in[0, T] \times D \\ \frac{\partial X}{\partial n_{\text {out }}}(t, x)=0, \quad \text { for } x \in \partial D \\ X(0, x) \geq 0 \text { and } 0<\mathbf{s}(0, x)+\mathbf{i}(0, x)+\mathbf{r}(0, x) \leq M .\end{array}\right.$

Writing the solution of (1.6) in its mild semigroup form, we have $X(t)=\widetilde{\mathrm{T}}(t) X(0)+\int_{0}^{t} \widetilde{\mathrm{T}}(t-r) G(X(r)) d r$, where we used the notation $\widetilde{\mathrm{T}}(t) X:=\left(\begin{array}{c}\mathrm{T}_{S}(t) \mathbf{s} \\ \mathrm{T}_{I}(t) \mathbf{i} \\ \mathrm{T}_{R}(t) \mathbf{r}\end{array}\right)$ and similarly for $\widetilde{\mathrm{T}}(t-r) G(X(r))$.

Lemma 1.2 The initial value probem (1.6) has a unique solution $X \in C\left([0, T] ;\left(L^{\infty}(D)\right)^{3}\right)$.

Proof : For $0 \leq u(0,) \leq$.1 , we define a mapping F : $C\left([0, T] ;\left(L^{\infty}(D)\right)^{3}\right) \longrightarrow C\left([0, T] ;\left(L^{\infty}(D)\right)^{3}\right)$ by

$$
(F u)(t)=\widetilde{\mathrm{T}}(t) u(0)+\int_{0}^{t} \widetilde{\mathrm{T}}(t-r) G(u(r)) d r
$$

Let $u, v \in C\left([0, T] ;\left(L^{\infty}(D)\right)^{3}\right)$ such that $u(0)=v(0)$. Using the fact that $\widetilde{T}$ is a contraction semigroup 
on $\left(L^{\infty}(D)\right)^{3}$ and $\mathrm{G}$ is globally Lipschitz, it follows that

$$
\|(F u)(t)-(F v)(t)\|_{\infty} \leq C \int_{0}^{t}\|u(r)-v(r)\|_{\infty} d r, \text { for all } t \in[0, T]
$$

where $C$ is the Lipschitz constant of $G$. Then we have

$$
\|(F u)(t)-(F v)(t)\|_{\infty} \leq C T \sup _{0 \leq r \leq T}\|u(r)-v(r)\|_{\infty}
$$

Using (1.7), (1.8) and induction on $n$, it follows that

$$
\sup _{0 \leq t \leq T}\left\|\left(F^{n} u\right)(t)-\left(F^{n} v\right)(t)\right\|_{\infty} \leq \frac{(C T)^{n}}{n !} \sup _{0 \leq t \leq T}\|u(t)-v(t)\|_{\infty} .
$$

For $n$ large enough $F^{n}$ is a contraction (since $\frac{(C T)^{n}}{n !}<1$ ). It follows from the Banach contraction principle that $\mathrm{F}$ has a unique fixed point in $X \in C\left([0, T] ;\left(L^{\infty}(D)\right)^{3}\right)$. This fixed point is the solution of

$$
X(t)=\widetilde{\mathrm{T}}(t) X(0)+\int_{0}^{t} \widetilde{\mathrm{T}}(t-r) G(X(r)) d r
$$

The mild solution of (1.10) is in fact a classical solution of (1.6), see Theorem 3.1, Chapter 7 of Smith (1995). Note that the Corollary 3.1, Chapter 7 of the above reference ensures that $X(t) \geq 0$, $\forall t \geq 0$.

\subsubsection{Relation between the system of PDEs and its discretisation}

We will now prove that $\mathcal{X}_{\varepsilon}$ converges to $\mathrm{X}$ as the mesh size $\varepsilon$ of the grid tends to zero.

Theorem 1.1 Let us consider an initial condition $X(0) \in\left(L^{\infty}(D)\right)^{3}$. For all $T>0$, $\sup _{t \in[0, T]}\left\|\mathcal{X}_{\varepsilon}(t)-X(t)\right\|_{\infty} \longrightarrow 0$, as $\varepsilon \rightarrow 0$.

Proof : Using the variation of constants formula, we have

$$
\begin{gathered}
\mathcal{S}_{\mathcal{\varepsilon}}(t)=\mathrm{T}_{\varepsilon, S}(t) \mathcal{S}_{\varepsilon}(0)-\int_{0}^{t} \mathrm{~T}_{\varepsilon, S}(t-r)\left[\frac{\beta_{\varepsilon}(.) \mathcal{S}_{\varepsilon}(r) \mathcal{I}_{\varepsilon}(r)}{\mathcal{S}_{\varepsilon}(r)+\mathcal{I}_{\varepsilon}(r)+\mathcal{R}_{\varepsilon}(r)}\right] d r \\
\mathbf{s}(t)=\mathrm{T}_{S}(t) \mathbf{s}(0)-\int_{0}^{t} \mathrm{~T}_{S}(t-r)\left[\frac{\beta(.) \mathbf{s}(r) \mathbf{i}(r)}{\mathbf{s}(r)+\mathbf{i}(r)+\mathbf{r}(r)}\right] d r .
\end{gathered}
$$

Recall that $\mathcal{S}_{\varepsilon}(0)=P_{\varepsilon} \mathbf{s}(0)$, so that

$$
\begin{aligned}
\mathcal{S}_{\mathcal{E}}(t)-\mathbf{s}(t)= & \mathrm{T}_{\mathcal{\varepsilon}, S}(t) P_{\varepsilon} \mathbf{s}(0)-\mathrm{T}_{S}(t) \mathbf{s}(0)-\int_{0}^{t} \mathrm{~T}_{\varepsilon, S}(t-r)\left[\frac{\beta_{\varepsilon}(.) \mathcal{S}_{\varepsilon}(r) \mathcal{I}_{\varepsilon}(r)}{\mathcal{S}_{\varepsilon}(r)+\mathcal{I}_{\varepsilon}(r)+\mathcal{R}_{\varepsilon}(r)}\right] d r \\
& +\int_{0}^{t} \mathrm{~T}_{S}(t-r)\left[\frac{\beta(.) \mathbf{s}(r) \mathbf{i}(r)}{\mathbf{s}(r)+\mathbf{i}(r)+\mathbf{r}(r)}\right] d r .
\end{aligned}
$$


We have

$$
\begin{aligned}
\| \mathcal{S}_{\mathcal{E}}(t)- & \mathbf{s}(t)\left\|_{\infty} \leq\right\| \mathrm{T}_{\varepsilon, S}(t) P_{\varepsilon} \mathbf{s}(0)-\mathrm{T}_{S}(t) \mathbf{s}(0) \|_{\infty} \\
& +\int_{0}^{t}\left\|\mathrm{~T}_{\varepsilon, S}(t-r)\left[\frac{\beta_{\varepsilon}(.) \mathcal{S}_{\varepsilon}(r) \mathcal{I}_{\varepsilon}(r)}{\mathcal{S}_{\varepsilon}(r)+\mathcal{I}_{\varepsilon}(r)+\mathcal{R}_{\varepsilon}(r)}\right]-\mathrm{T}_{S}(t-r)\left[\frac{\beta(.) \mathbf{s}(r) \mathbf{i}(r)}{\mathbf{s}(r)+\mathbf{i}(r)+\mathbf{r}(r)}\right]\right\|_{\infty} d r \\
\leq & \left\|\mathrm{T}_{\varepsilon, S}(t) P_{\varepsilon} \mathbf{s}(0)-\mathrm{T}_{S}(t) \mathbf{s}(0)\right\|_{\infty} \\
& +\int_{0}^{t}\left\|\mathrm{~T}_{\varepsilon, S}(t-r)\left[\frac{\beta \varepsilon(.) \mathcal{S}_{\varepsilon}(r) \mathcal{I}_{\varepsilon}(r)}{\mathcal{S}_{\varepsilon}(r)+\mathcal{I}_{\varepsilon}(r)+\mathcal{R}_{\varepsilon}(r)}\right]-\mathrm{T}_{\varepsilon, S}(t-r) P_{\varepsilon}\left[\frac{\beta(.) \mathbf{s}(r) \mathbf{i}(r)}{\mathbf{s}(r)+\mathbf{i}(r)+\mathbf{r}(r)}\right]\right\|_{\infty} d r \\
& +\int_{0}^{t}\left\|\mathrm{~T}_{\varepsilon, S}(t-r) P_{\varepsilon}\left[\frac{\beta(.) \mathbf{s}(r) \mathbf{i}(r)}{\mathbf{s}(r)+\mathbf{i}(r)+\mathbf{r}(r)}\right]-\mathrm{T}_{S}(t-r)\left[\frac{\beta(.) \mathbf{s}(r) \mathbf{i}(r)}{\mathbf{s}(r)+\mathbf{i}(r)+\mathbf{r}(r)}\right]\right\|_{\infty} d r .
\end{aligned}
$$

Let us estimate each term of the right-hand side of this inequality.

Since $\mathbf{s}(0) \in L^{\infty}(D)$, it then follows from Kato ( [12] pp. 512-513), that

$$
\left\|\mathrm{T}_{\varepsilon, S}(t) P_{\varepsilon} \mathbf{s}(0)-\mathrm{T}_{S}(t) \mathbf{s}(0)\right\|_{\infty} \longrightarrow 0, \text { uniformly on }[0, T] .
$$

Using the fact that $\mathrm{T}_{\varepsilon, S}$ is a contraction semigroup on $\left(H^{\varepsilon},\|\cdot\|_{\infty}\right)$, we obtain

$$
\begin{aligned}
\int_{0}^{t}\left\|\mathrm{~T}_{\varepsilon, S}(t-r)\left[\frac{\beta_{\varepsilon}(.) \mathcal{S}_{\varepsilon}(r) \mathcal{I}_{\varepsilon}(r)}{\mathcal{S}_{\varepsilon}(r)+\mathcal{I}_{\varepsilon}(r)+\mathcal{R}_{\varepsilon}(r)}\right]-\mathrm{T}_{\varepsilon, S}(t-r) P_{\varepsilon}\left[\frac{\beta(.) \mathbf{s}(r) \mathbf{i}(r)}{\mathbf{s}(r)+\mathbf{i}(r)+\mathbf{r}(r)}\right]\right\|_{\infty} d r \\
\leq \bar{\beta} \int_{0}^{t}\left\|\frac{\mathcal{S}_{\varepsilon}(r) \mathcal{I}_{\varepsilon}(r)}{\mathcal{S}_{\varepsilon}(r)+\mathcal{I}_{\varepsilon}(r)+\mathcal{R}_{\varepsilon}(r)}-\frac{\mathbf{s}(r) \mathbf{i}(r)}{\mathbf{s}(r)+\mathbf{i}(r)+\mathbf{r}(r)}\right\|_{\infty} d r \\
+\int_{0}^{t}\left\|P_{\varepsilon}\left[\frac{\beta(.) \mathbf{s}(r) \mathbf{i}(r)}{\mathbf{s}(r)+\mathbf{i}(r)+\mathbf{r}(r)}\right]-\frac{\beta(.) \mathbf{s}(r) \mathbf{i}(r)}{\mathbf{s}(r)+\mathbf{i}(r)+\mathbf{r}(r)}\right\| \|_{\infty} d r \\
\leq \bar{\beta} \int_{0}^{t}\left(2\left\|\mathcal{S}_{\varepsilon}(r)-\mathbf{s}(r)\right\|_{\infty}+2\left\|\mathcal{I}_{\varepsilon}(r)-\mathbf{i}(r)\right\|_{\infty}+\left\|\mathcal{R}_{\varepsilon}(r)-\mathbf{r}(r)\right\|_{\infty}\right) d r \\
+\int_{0}^{t}\left\|P_{\varepsilon}\left[\frac{\beta(.) \mathbf{s}(r) \mathbf{i}(r)}{\mathbf{s}(r)+\mathbf{i}(r)+\mathbf{r}(r)}\right]-\frac{\beta(.) \mathbf{s}(r) \mathbf{i}(r)}{\mathbf{s}(r)+\mathbf{i}(r)+\mathbf{r}(r)}\right\| \|_{\infty} d r .
\end{aligned}
$$

Since $\frac{\beta(.) \mathbf{s}(r) \mathbf{i}(r)}{\mathbf{s}(r)+\mathbf{i}(r)+\mathbf{r}(r)} \in L^{\infty}(D)$, then for the last term (1.11), we have

$$
\left\|\mathrm{T}_{\varepsilon, S}(t-r) P_{\varepsilon}\left[\frac{\beta(.) \mathbf{s}(r) \mathbf{i}(r)}{\mathbf{s}(r)+\mathbf{i}(r)+\mathbf{r}(r)}\right]-\mathrm{T}_{S}(t-r)\left[\frac{\beta(.) \mathbf{s}(r) \mathbf{i}(r)}{\mathbf{s}(r)+\mathbf{i}(r)+\mathbf{r}(r)}\right]\right\|_{\infty} \longrightarrow 0, \text { uniformly on }[0, T],
$$

( thanks by Kato [12], chapter 9, Section 3 ). Consequently

$$
\begin{aligned}
& \int_{0}^{t}\left\|\mathrm{~T}_{\varepsilon, S}(t-r) P_{\varepsilon}\left[\frac{\beta(.) \mathbf{s}(r) \mathbf{i}(r)}{\mathbf{s}(r)+\mathbf{i}(r)+\mathbf{r}(r)}\right]-\mathrm{T}_{S}(t-r)\left[\frac{\beta(.) \mathbf{s}(r) \mathbf{i}(r)}{\mathbf{s}(r)+\mathbf{i}(r)+\mathbf{r}(r)}\right]\right\|_{\infty} d r \longrightarrow 0 . \\
& \quad\left\|\mathcal{S}_{\mathcal{E}}(t)-\mathbf{s}(t)\right\|_{\infty} \leq a_{\mathcal{\varepsilon}}(t)+C(\bar{\beta}) \int_{0}^{t}\left(\left\|\mathcal{S}_{\mathcal{\varepsilon}}(r)-\mathbf{s}(r)\right\|_{\infty}+\left\|\mathcal{I}_{\mathcal{\varepsilon}}(r)-\mathbf{i}(r)+\right\| \mathcal{R}_{\mathcal{\varepsilon}}(r)-\mathbf{r}(r) \|_{\infty}\right) d r, \text { where }
\end{aligned}
$$




$$
\begin{aligned}
a_{\varepsilon}(t)= & \left\|\mathrm{T}_{\varepsilon, S}(t) P_{\varepsilon} \mathbf{s}(0)-\mathrm{T}_{S}(t) \mathbf{s}(0)\right\|_{\infty}+\int_{0}^{t}\left\|P_{\varepsilon}\left[\frac{\beta(.) \mathbf{s}(r) \mathbf{i}(r)}{\mathbf{s}(r)+\mathbf{i}(r)+\mathbf{r}(r)}\right]-\frac{\beta(.) \mathbf{s}(r) \mathbf{i}(r)}{\mathbf{s}(r)+\mathbf{i}(r)+\mathbf{r}(r)}\right\|_{\infty} d r \\
& +\int_{0}^{t}\left\|\mathrm{~T}_{\varepsilon, S}(t-r) P_{\varepsilon}\left[\frac{\beta(.) \mathbf{s}(r) \mathbf{i}(r)}{\mathbf{s}(r)+\mathbf{i}(r)+\mathbf{r}(r)}\right]-\mathrm{T}_{S}(t-r)\left[\frac{\beta(.) \mathbf{s}(r) \mathbf{i}(r)}{\mathbf{s}(r)+\mathbf{i}(r)+\mathbf{r}(r)}\right]\right\|_{\infty} d r,
\end{aligned}
$$

Exactly in the same way we have a similar inequality for $\left\|\mathcal{I}_{\varepsilon}(t)-\mathbf{i}(t)\right\|_{\infty}$ and $\left\|\mathcal{R}_{\varepsilon}(t)-\mathbf{r}(t)\right\|_{\infty}$ with $\mathrm{T}_{I}$, $\mathrm{T}_{R}$ in place of $\mathrm{T}_{S}$, and $\mathrm{T}_{\varepsilon, I}, \mathrm{~T}_{\varepsilon, R}$ in place of $\mathrm{T}_{\varepsilon, S}$, respectively. Combining those estimates we obtain

$$
\left\|\mathcal{X}_{\varepsilon}(t)-X(t)\right\|_{\infty} \leq \tilde{a}_{\varepsilon}(t)+C(\bar{\alpha}, \bar{\beta}) \int_{0}^{t}\left\|\mathcal{X}_{\varepsilon}(r)-X(r)\right\|_{\infty} d r
$$

where $\sup _{0 \leq t \leq T} \tilde{a}_{\varepsilon}(t) \longrightarrow 0$, as $\varepsilon \rightarrow 0$. Applying Gronwall's Lemma, it follows that

$$
\sup _{0 \leq t \leq T}\left\|\mathcal{X}_{\varepsilon}(t)-X(t)\right\|_{\infty} \leq \sup _{0 \leq t \leq T} \tilde{a}_{\varepsilon}(t) e^{C(\bar{\alpha}, \bar{\beta}) T}
$$

Finally, the theorem follows from the fact that the right-hand side tends to zero as $\varepsilon \rightarrow 0$.

\subsection{The stochastic model}

Deterministic models describe the spread of disease under the assumptions of mass action, relying on the law of large numbers. The most natural way to describe the spread of disease is stochastic. The previous models are based on the hypothesis of a population of large size. When it is not the case, the interactions between the individuals are not uniform but possess an intrinsic random character. We are going to expose now a probabilistic version of the previous model. For each given site, Poisson processes count the number of new infections, removal and migrations between sites during time. So the propagation of 
the illness can be modeled by the following system of stochastic differential equations

$$
\left\{\begin{aligned}
S^{\varepsilon}\left(t, x_{i}\right) & =S^{\varepsilon}\left(0, x_{i}\right)-\mathrm{P}_{x_{i}}^{i n f}\left(\int_{0}^{t} \frac{\beta\left(x_{i}\right) S^{\varepsilon}\left(r, x_{i}\right) I^{\varepsilon}\left(r, x_{i}\right)}{S^{\varepsilon}\left(r, x_{i}\right)+I^{\varepsilon}\left(r, x_{i}\right)+R^{\varepsilon}\left(r, x_{i}\right)} d r\right) \\
& -\sum_{y_{i} \sim x_{i}} \mathrm{P}_{S, x_{i}, y_{i}}^{m i g}\left(\int_{0}^{t} \frac{\mu_{S}}{\varepsilon^{2}} S^{\varepsilon}\left(r, x_{i}\right) d r\right)+\sum_{y_{i} \sim x_{i}} \mathrm{P}_{S, y_{i}, x_{i}}^{m i g}\left(\int_{0}^{t} \frac{\mu_{S}}{\varepsilon^{2}} S^{\varepsilon}\left(r, y_{i}\right) d r\right) \\
I^{\varepsilon}\left(t, x_{i}\right) & =I^{\varepsilon}\left(0, x_{i}\right)+\mathrm{P}_{x_{i}}^{i n f}\left(\int_{0}^{t} \frac{\beta\left(x_{i}\right) S^{\varepsilon}\left(r, x_{i}\right) I^{\varepsilon}\left(r, x_{i}\right)}{S^{\varepsilon}\left(r, x_{i}\right)+I^{\varepsilon}\left(r, x_{i}\right)+R^{\varepsilon}\left(r, x_{i}\right)} d r\right)-\mathrm{P}_{x_{i}}^{r e c}\left(\int_{0}^{t} \alpha\left(x_{i}\right) I^{\varepsilon}\left(r, x_{i}\right) d r\right) \\
& -\sum_{y_{i} \sim x_{i}} \mathrm{P}_{I, x_{i}, y_{i}}^{m i g}\left(\int_{0}^{t} \frac{\mu_{I}}{\varepsilon^{2}} I^{\varepsilon}\left(r, x_{i}\right) d r\right)+\sum_{y_{i} \sim x_{i}} \mathrm{P}_{I, y_{i}, x_{i}}^{m i g}\left(\int_{0}^{t} \frac{\mu_{I}}{\varepsilon^{2}} I^{\varepsilon}\left(r, y_{i}\right) d r\right) \\
R^{\varepsilon}\left(t, x_{i}\right) & =R^{\varepsilon}\left(0, x_{i}\right)+\mathrm{P}_{x_{i}}^{r e c}\left(\int_{0}^{t} \alpha\left(x_{i}\right) I^{\varepsilon}\left(r, x_{i}\right) d r\right)-\sum_{y_{i} \sim x_{i}} \mathrm{P}_{R, x_{i}, y_{i}}^{m i g}\left(\int_{0}^{t} \frac{\mu_{R}}{\varepsilon^{2}} R^{\varepsilon}\left(r, x_{i}\right) d r\right) \\
& +\sum_{y_{i} \sim x_{i}} \mathrm{P}_{R, y_{i}, x_{i}}^{m i g}\left(\int_{0}^{t} \frac{\mu_{R}}{\varepsilon^{2}} R^{\varepsilon}\left(r, y_{i}\right) d r\right), \quad\left(t, x_{i}\right) \in[0, T] \times D_{\varepsilon},
\end{aligned}\right.
$$

where all the $\mathrm{P}_{j}$ 's are mutually independent standard Poisson processes. In this system, at a given site $x_{i}$

- infection of a susceptible happens at rate $\beta\left(x_{i}\right) \frac{S^{\varepsilon}\left(t, x_{i}\right)}{S^{\varepsilon}\left(t, x_{i}\right)+I^{\varepsilon}\left(t, x_{i}\right)+R^{\varepsilon}\left(t, x_{i}\right)} I^{\varepsilon}\left(t, x_{i}\right)$. Then $\mathrm{P}_{x_{i}}^{\text {inf }}\left(\int_{0}^{t} \frac{\beta\left(x_{i}\right) S^{\varepsilon}\left(r, x_{i}\right) I^{\varepsilon}\left(r, x_{i}\right)}{S^{\varepsilon}\left(r, x_{i}\right)+I^{\varepsilon}\left(r, x_{i}\right)+R^{\varepsilon}\left(r, x_{i}\right)} d r\right)$ counts the number of transitions of type $S^{\varepsilon} \longrightarrow I^{\varepsilon}$ at site $x_{i}$ between time 0 and time $t$.

- recovery of an infectious happens at rate $\alpha\left(x_{i}\right) I^{\varepsilon}\left(t, x_{i}\right)$, so $\mathrm{P}_{x_{i}}^{r e c}\left(\int_{0}^{t} \alpha\left(x_{i}\right) I^{\varepsilon}\left(r, x_{i}\right) d r\right)$ counts the number of transitions of type $I^{\varepsilon} \longrightarrow R^{\varepsilon}$ at site $x_{i}$ between time 0 and time $t$.

- The term $\mathrm{P}_{S, x_{i}, y_{i}}^{m i g}\left(\int_{0}^{t} \frac{\mu_{S}}{\varepsilon^{2}} S^{\varepsilon}\left(r, x_{i}\right) d r\right)$ counts the number of migrations of susceptibles from site $x_{i}$ to $y_{i}$ (where $x_{i}$ and $y_{i}$ are neighbors), those events happen at rate $\frac{\mu_{S}}{\varepsilon^{2}} S^{\varepsilon}\left(t, x_{i}\right)$; and similarly for the compartments $I^{\varepsilon}$ and $R^{\varepsilon}$.

We introduce the martingales $\mathrm{M}_{j}(t)=\mathrm{P}_{j}(t)-t$ and we look instead at the renormalized model by dividing the number of individuals in each compartment and at each site by $\mathbf{N}$. Hence by setting

$$
S_{\mathbf{N}, \varepsilon}\left(t, x_{i}\right)=\frac{S^{\varepsilon}\left(t, x_{i}\right)}{\mathbf{N}}, I_{\mathbf{N}, \varepsilon}\left(t, x_{i}\right)=\frac{I^{\varepsilon}\left(t, x_{i}\right)}{\mathbf{N}}, \text { and } R_{\mathbf{N}, \varepsilon}\left(t, x_{i}\right)=\frac{R^{\varepsilon}\left(t, x_{i}\right)}{\mathbf{N}}
$$


the equations in the various compartments read

$$
\left\{\begin{array}{l}
S_{\mathbf{N}, \varepsilon}\left(t, x_{i}\right)=S_{\mathbf{N}, \varepsilon}\left(0, x_{i}\right)-\int_{0}^{t} \frac{\beta\left(x_{i}\right) S_{\mathbf{N}, \varepsilon}\left(r, x_{i}\right) I_{\mathbf{N}, \varepsilon}\left(r, x_{i}\right)}{S_{\mathbf{N}, \varepsilon}\left(r, x_{i}\right)+I_{\mathbf{N}, \varepsilon}\left(r, x_{i}\right)+R_{\mathbf{N}, \varepsilon}\left(r, x_{i}\right)} d r \\
+\int_{0}^{t} \mu_{S} \Delta_{\varepsilon} S_{\mathbf{N}, \varepsilon}\left(r, x_{i}\right) d r-\frac{1}{\mathbf{N}} \mathbf{M}_{x_{i}}^{i n f}\left(\mathbf{N} \int_{0}^{t} \frac{\beta\left(x_{i}\right) S_{\mathbf{N}, \varepsilon}\left(r, x_{i}\right) I_{\mathbf{N}, \varepsilon}\left(r, x_{i}\right)}{S_{\mathbf{N}, \varepsilon}\left(r, x_{i}\right)+I_{\mathbf{N}, \varepsilon}\left(r, x_{i}\right)+R_{\mathbf{N}, \varepsilon}\left(r, x_{i}\right)} d r\right) \\
-\sum_{y_{i} \sim x_{i}} \frac{1}{\mathbf{N}} \mathbf{M}_{S, x_{i}, y_{i}}^{m i g}\left(\mathbf{N} \int_{0}^{t} \frac{\mu_{S}}{\varepsilon^{2}} S_{\mathbf{N}, \varepsilon}\left(r, x_{i}\right) d r\right)+\sum_{y_{i} \sim x_{i}} \frac{1}{\mathbf{N}} \mathbf{M}_{S, y_{i}, x_{i}}^{m i g}\left(\mathbf{N} \int_{0}^{t} \frac{\mu_{S}}{\varepsilon^{2}} S_{\mathbf{N}, \varepsilon}\left(r, y_{i}\right) d r\right) \\
I_{\mathbf{N}, \varepsilon}\left(t, x_{i}\right)=I_{\mathbf{N}, \varepsilon}\left(0, x_{i}\right)+\int_{0}^{t} \frac{\beta\left(x_{i}\right) S_{\mathbf{N}, \varepsilon}\left(r, x_{i}\right) I_{\mathbf{N}, \varepsilon}\left(r, x_{i}\right)}{S_{\mathbf{N}, \varepsilon}\left(r, x_{i}\right)+I_{\mathbf{N}, \varepsilon}\left(r, x_{i}\right)+R_{\mathbf{N}, \varepsilon}\left(r, x_{i}\right)} d r-\int_{0}^{t} \alpha\left(x_{i}\right) I_{\mathbf{N}, \varepsilon}\left(r, x_{i}\right) d r \\
+\mu_{I} \int_{0}^{t} \Delta_{\varepsilon} I_{\mathbf{N}, \varepsilon}\left(r, x_{i}\right) d r+\frac{1}{\mathbf{N}} \mathbf{M}_{x_{i}}^{i n f}\left(\mathbf{N} \int_{0}^{t} \frac{\beta\left(x_{i}\right) S_{\mathbf{N}, \varepsilon}\left(r, x_{i}\right) I_{\mathbf{N}, \varepsilon}\left(r, x_{i}\right)}{S_{\mathbf{N}, \varepsilon}\left(r, x_{i}\right)+I_{\mathbf{N}, \varepsilon}\left(r, x_{i}\right)+R_{\mathbf{N}, \varepsilon}\left(r, x_{i}\right)} d r\right) \\
-\frac{1}{\mathbf{N}} \mathbf{M}_{x_{i}}^{r e c}\left(\mathbf{N} \int_{0}^{t} \alpha\left(x_{i}\right) I_{\mathbf{N}, \varepsilon}\left(r, x_{i}\right) d r\right)-\sum_{y_{i} \sim x_{i}} \frac{1}{\mathbf{N}} \mathbf{M}_{I, x_{i}, y_{i}}^{m i g}\left(\mathbf{N} \int_{0}^{t} \frac{\mu_{I}}{\varepsilon^{2}} I_{\mathbf{N}, \varepsilon}\left(r, x_{i}\right) d r\right) \\
+\sum_{y_{i} \sim x_{i}} \frac{1}{\mathbf{N}} \mathbf{M}_{I, y_{i}, x_{i}}^{m i g}\left(\mathbf{N} \int_{0}^{t} \frac{\mu_{I}}{\varepsilon^{2}} I_{\mathbf{N}, \varepsilon}\left(r, y_{i}\right) d r\right) \\
R_{\mathbf{N}, \varepsilon}\left(t, x_{i}\right)=R_{\mathbf{N}, \varepsilon}\left(0, x_{i}\right)+\int_{0}^{t} \alpha\left(x_{i}\right) I_{\mathbf{N}, \varepsilon}\left(r, x_{i}\right) d r+\int_{0}^{t} \mu_{R} \Delta_{\varepsilon} R_{\mathbf{N}, \varepsilon}\left(r, x_{i}\right) d r \\
+\frac{1}{\mathbf{N}} \mathbf{M}_{x_{i}}^{r e c}\left(\mathbf{N} \int_{0}^{t} \alpha\left(x_{i}\right) I_{\mathbf{N}, \varepsilon}\left(r, x_{i}\right) d r\right)-\sum_{y_{i} \sim x_{i}} \frac{1}{\mathbf{N}} \mathbf{M}_{R, x_{i}, y_{i}}^{m i g}\left(\mathbf{N} \int_{0}^{t} \frac{\mu_{R}}{\varepsilon^{2}} R_{\mathbf{N}, \varepsilon}\left(r, x_{i}\right) d r\right) \\
+\sum_{y_{i} \sim x_{i}} \frac{1}{\mathbf{N}} \mathbf{M}_{R, y_{i}, x_{i}}^{m i g}\left(\mathbf{N} \int_{0}^{t} \frac{\mu_{R}}{\varepsilon^{2}} R_{\mathbf{N}, \varepsilon}\left(r, y_{i}\right) d r\right) .
\end{array}\right.
$$

Let $S_{\mathbf{N}, \varepsilon}(t)$ and $I_{\mathbf{N}, \varepsilon}(t)$ and $R_{\mathbf{N}, \varepsilon}(t)$ denote respectively the vectors which describe the "proportions" of susceptibles, infectious and removed in the population at the various sites at time $t$ :

$S_{\mathbf{N}, \varepsilon}(t)=\left(\begin{array}{c}S_{\mathbf{N}, \varepsilon}\left(t, x_{1}\right) \\ \vdots \\ S_{\mathbf{N}, \varepsilon}\left(t, x_{\ell}\right)\end{array}\right), \quad I_{\mathbf{N}, \varepsilon}(t)=\left(\begin{array}{c}I_{\mathbf{N}, \varepsilon}\left(t, x_{1}\right) \\ \vdots \\ I_{\mathbf{N}, \varepsilon}\left(t, x_{\ell}\right)\end{array}\right)$ and $\quad R_{\mathbf{N}, \varepsilon}(t)=\left(\begin{array}{c}R_{\mathbf{N}, \varepsilon}\left(t, x_{1}\right) \\ \vdots \\ R_{\mathbf{N}, \varepsilon}\left(t, x_{\ell}\right)\end{array}\right)$

where $\ell$ is the total number of locations. Let us set $Z_{\mathbf{N}, \varepsilon}(t)=\left(\begin{array}{c}S_{\mathbf{N}, \varepsilon}(t) \\ I_{\mathbf{N}, \varepsilon}(t) \\ R_{\mathbf{N}, \varepsilon}(t)\end{array}\right)$; then the aggregated form of the stochastic model is

(1.15) $Z_{\mathbf{N}, \varepsilon}(t)=Z_{\mathbf{N}, \varepsilon}(0)+\int_{0}^{t} b_{\varepsilon}\left(r, Z_{\mathbf{N}, \varepsilon}(r)\right) d r+\sum_{j=1}^{k_{\varepsilon}} \frac{h_{j}}{\mathbf{N}} M_{j}\left(\mathbf{N} \int_{0}^{t} \beta_{j}\left(r, Z_{\mathbf{N}, \varepsilon}(r)\right) d r\right)$,

where $\forall r \geq 0, b_{\varepsilon}\left(r, Z_{\mathbf{N}, \varepsilon}(r)\right)=\sum_{j=1}^{k_{\varepsilon}} h_{j} \beta_{j}\left(r, Z_{\mathbf{N}, \varepsilon}(r)\right)$; the coordinates of each vector $h_{j}$ are either $-1,0$ or 1 and 
$\beta_{j}\left(r, Z_{\mathbf{N}, \varepsilon}(r)\right) \in\left\{\frac{\beta(.) S_{\mathbf{N}, \varepsilon}\left(r, x_{i}\right) I_{\mathbf{N}, \varepsilon}(r, .)}{S_{\mathbf{N}, \varepsilon}(r, .)+I_{\mathbf{N}, \varepsilon}(r, .)+R_{\mathbf{N}, \varepsilon}(r, .)}, \frac{\mu_{S}}{\varepsilon^{2}} S_{\mathbf{N}, \varepsilon}(r,),. \frac{\mu_{I}}{\varepsilon^{2}} I_{\mathbf{N}, \varepsilon}(r,),. \frac{\mu_{R}}{\varepsilon^{2}} R_{\mathbf{N}, \varepsilon}(r,),. \alpha(.) I_{\mathbf{N}, \varepsilon}(r,).\right\}$,

$k_{\varepsilon}$ is the total number of Poisson processes in the model. Note that $b_{\varepsilon}\left(r, Z_{\mathbf{N}, \varepsilon}(r)\right)=\widetilde{\Delta}_{\varepsilon} Z_{\mathbf{N}, \varepsilon}(r)+G\left(Z_{\mathbf{N}, \varepsilon}(r)\right)$, where

$$
\widetilde{\Delta}_{\varepsilon} Z_{\mathbf{N}, \varepsilon}(r)=\left(\begin{array}{l}
\mu_{S} \Delta_{\varepsilon} S_{\mathbf{N}, \varepsilon}(r) \\
\mu_{I} \Delta_{\varepsilon} I_{\mathbf{N}, \varepsilon}(r) \\
\mu_{R} \Delta_{\varepsilon} R_{\mathbf{N}, \varepsilon}(r)
\end{array}\right) \text { and } G\left(Z_{\mathbf{N}, \varepsilon}(r)\right)=\left(\begin{array}{l}
-\frac{\beta(.) S_{\mathbf{N}, \varepsilon}(r) I_{\mathbf{N}, \varepsilon}(r)}{S_{\mathbf{N}, \varepsilon}(r)+I_{\mathbf{N}, \varepsilon}\left(r, x_{i}\right)+I_{\mathbf{N}, \varepsilon}(r)} \\
\frac{\beta(.) S_{\mathbf{N}, \varepsilon}(r) I_{\mathbf{N}, \varepsilon}(r)}{S_{\mathbf{N}, \varepsilon}(r)+I_{\mathbf{N}, \varepsilon}(r)+I_{\mathbf{N}, \varepsilon}(r)}-\alpha(.) I_{\mathbf{N}, \varepsilon}(r) \\
\alpha(.) I_{\mathbf{N}, \varepsilon}(r)
\end{array}\right) .
$$

\section{Existence and uniqueness}

At the begining of the epidemic, the proportions of the population in various compartments take their values in the discrete set $\left\{\frac{n}{\mathbf{N}}, n=0,1, \cdots\right\}$, and since the Poisson processes are mutually independent, this implies that the components of $Z_{\mathbf{N}, \varepsilon}(t)$ remain non-negative for all $t \geq 0$. Indeed, let us consider for example the component $S_{\mathbf{N}, \varepsilon}$. Since all jumps of each $S_{\mathbf{N}, \varepsilon}\left(t, x_{i}\right)$ are of size $\pm \frac{1}{\mathbf{N}}$, before becoming negative, $S_{\mathbf{N}, \varepsilon}\left(t, x_{i}\right)$ is zero. But as long as $S_{\mathbf{N}, \varepsilon}\left(t, x_{i}\right)=0$, the rate of its negative jumps is zero, hence $S_{\mathbf{N}, \varepsilon}\left(t, x_{i}\right)$ cannot become negative.

$\sum_{i=1}^{\varepsilon^{-d}}\left(S_{\mathbf{N}, \varepsilon}\left(t, x_{i}\right)+I_{\mathbf{N}, \varepsilon}\left(t, x_{i}\right)+R_{\mathbf{N}, \varepsilon}\left(t, x_{i}\right)\right)=\varepsilon^{-d}$, since this quantity does not depend upon $t$. It then follows that $0 \leq Z_{\mathbf{N}, \varepsilon}(t) \leq \varepsilon^{-d}$, for all $t \geq 0$. Then by letting $\beta_{T}^{\mathbf{N}, \varepsilon}=\sup _{\substack{1 \leq j \leq \ell \\ 0 \leq t \leq T}} \beta_{j}\left(t, Z_{\mathbf{N}, \varepsilon}(t)\right)$, we have that $\beta_{T}^{\mathbf{N}, \varepsilon} \leq \bar{C}$, where $\bar{C}=\max \left\{\bar{\beta}, \bar{\alpha}, \frac{\mu_{S}}{\varepsilon^{2}}, \frac{\mu_{I}}{\varepsilon^{2}}, \frac{\mu_{R}}{\varepsilon^{2}}, \varepsilon^{-d}\right\}$

$$
Z_{\mathbf{N}, \varepsilon}(t)=Z_{\mathbf{N}, \varepsilon}(0)+\sum_{j=1}^{k_{\varepsilon}} \frac{h_{j}}{\mathbf{N}} P_{j}\left(\mathbf{N} \int_{0}^{t} \beta_{j}\left(r, Z_{\mathbf{N}, \varepsilon}(r)\right) d r\right) .
$$

Let $\tau_{1}^{j}<\tau_{2}^{j}<\cdots$ be the jump times of the Poisson process $P_{j}(t), 1 \leq j \leq k$. As long as $\mathbf{N} \beta_{j}\left(Z_{\mathbf{N}, \varepsilon}(0)\right) \times t<\tau_{1}^{j}$, for all $1 \leq j \leq k$, the process $Z_{\mathbf{N}, \varepsilon}(t)$ remains constant. Let us set

$$
T_{1}=\inf \left\{t>0: \mathbf{N} \beta_{j}\left(Z_{\mathbf{N}, \varepsilon}(0)\right) \times t=\tau_{1}^{j} \text {, for some } 1 \leq j \leq k\right\} .
$$

The independence of the $P_{j}$ 's ensures that there is almost surely a unique $j_{0}$ such that $\mathbf{N} \beta_{j_{0}}\left(Z_{\mathbf{N}, \varepsilon}(0)\right) \times T_{1}=\tau_{1}^{j_{0}}$. In this case $Z_{\mathbf{N}, \varepsilon}\left(T_{1}\right)=Z_{\mathbf{N}, \varepsilon}(0)+\frac{h_{j_{0}}}{\mathbf{N}}$, and the process remains constant until 
the next jump of one of the $P_{j}$ 's. We wait for the next time for which one of the integrands

$$
\int_{0}^{t} \mathbf{N} \beta_{j}\left(Z_{\mathbf{N}, \varepsilon}(r)\right) d r=\mathbf{N} \beta_{j}\left(Z_{\mathbf{N}, \varepsilon}(0)\right) \times T_{1}+\mathbf{N} \beta_{j}\left(Z_{\mathbf{N}, \varepsilon}(0)+\frac{h_{j_{0}}}{\mathbf{N}}\right)\left(t-T_{1}\right)
$$

will be equal to the jump time of one of the $P_{j}$. We continue this procedure . Since there are a finite number of $P_{j}$ and the rates $\beta_{j}$ are bounded, any time $t \in[0, T]$ is achieved after a finite number of operations as above. This construction shows existence and uniqueness of the solution of (1.16).

\section{Law of large numbers ( $\mathbf{N} \rightarrow \infty, \varepsilon$ being fixed)}

Recall that, from Assumption 1.1, $\int_{D}(\mathbf{s}(0, x)+\mathbf{i}(0, x)+\mathbf{r}(0, x)) d x=1$. Recall that in the stochastic model, we have a total of $\mathbf{N} \varepsilon^{-d}$ individuals. At time $t=0$, each individual, independently of the others, is susceptible and located at site $x_{i}$ with probability $\int_{V_{i}} \mathbf{s}(0, x) d x$, infectious and located at site $x_{i}$ with probability $\int_{V_{i}} \mathbf{i}(0, x) d x$, removed and located at site $x_{i}$ with probability $\int_{V_{i}} \mathbf{r}(0, x) d x, 1 \leq i \leq \varepsilon^{-d}$. It follows from the choice of the initial condition of the stochastic system, the law of large numbers and the definition (1.13) that for any $1 \leq i \leq \varepsilon^{-d}$, as $N \rightarrow \infty, S_{\mathbf{N}, \varepsilon}\left(0, x_{i}\right) \longrightarrow \varepsilon^{-d} \int_{V_{i}} \mathbf{s}(0, x) d x, I_{\mathbf{N}, \varepsilon}\left(0, x_{i}\right) \longrightarrow$ $\varepsilon^{-d} \int_{V_{i}} \mathbf{i}(0, x) d x$ and $R_{\mathbf{N}, \varepsilon}\left(0, x_{i}\right) \longrightarrow \varepsilon^{-d} \int_{V_{i}} \mathbf{r}(0, x) d x$, a.s. .

In this section we fix the mesh size $\varepsilon$ of the grid and we let $\mathbf{N}$ go to infinity. We will show that the stochastic model converges to the corresponding deterministic model on the grid. First let us recall the law of large numbers for Poisson processes.

Lemma 2.1 Let $\{P(t), t \geq 0\}$ be a rate $\lambda$ Poisson process. Then

$$
\frac{P(t)}{t} \longrightarrow \lambda \text { a.s } \quad \text { as } t \rightarrow \infty \text {. }
$$

A proof of this well-known lemma can be found e.g. in Britton and Pardoux (2019).

In the sequel, we shall assume that $Z_{\mathbf{N}, \varepsilon}(t)$ is defined on the probability space $\left(\Omega, \mathcal{F}, \mathcal{F}_{t}^{\mathbf{N}, \varepsilon}, \mathbb{P}\right)$, where $\mathcal{F}_{t}^{\mathbf{N}, \varepsilon}=\sigma\left\{Z_{\mathbf{N}, \varepsilon}\left(r, x_{i}\right), 0 \leq r \leq t ; x_{i} \in D_{\varepsilon}\right\}$. If we consider the $k_{\varepsilon}$-dimensional process $\left(M_{\mathbf{N}, \varepsilon}^{j}\right)_{1 \leq j \leq k_{\varepsilon}}$ whose j-th component is defined as $M_{\mathbf{N}, \varepsilon}^{j}\left(t, x_{i}\right)=\frac{1}{\mathbf{N}} \mathbf{M}_{j}\left(\mathbf{N} \int_{0}^{t} \beta_{j}\left(Z_{\mathbf{N}, \varepsilon}\left(r, x_{i}\right)\right) d r\right)$, for a site $x_{i} \in D_{\varepsilon}$, then we have the following Proposition.

Proposition 2.1 For all $1 \leq j \leq k_{\varepsilon}$ for all $T>0$, as $\mathbf{N} \rightarrow+\infty$,

$$
\sup _{0 \leq t \leq T}\left|M_{\mathbf{N}, \varepsilon}^{j}\left(t, x_{i}\right)\right| \stackrel{\text { a.s. }}{\longrightarrow} 0
$$


Proof : For all $T>0$ we have

$$
\begin{aligned}
\sup _{0 \leq t \leq T}\left|M_{\mathbf{N}, \varepsilon}^{j}\left(t, x_{i}\right)\right| & =\sup _{0 \leq t \leq T}\left|\frac{1}{\mathbf{N}} M_{j}\left(\int_{0}^{t} \mathbf{N} \beta_{j}\left(Z_{\mathbf{N}, \varepsilon}\left(r, x_{i}\right)\right) d r\right)\right| \\
& \leq \sup _{0 \leq t \leq T \bar{C}}\left|\frac{1}{\mathbf{N}} M_{j}(\mathbf{N} t)\right| \quad\left(\text { because } 0 \leq \beta_{j} \leq \bar{C}\right) \\
& =\sup _{0 \leq t \leq T \bar{C}}\left|\frac{1}{\mathbf{N}} P_{j}(\mathbf{N} t)-t\right| .
\end{aligned}
$$

From Lemma 2.1,

$$
\frac{P_{j}(\mathbf{N} t)}{\mathbf{N}} \longrightarrow t \text { a.s., as } \mathbf{N} \rightarrow \infty .
$$

We have pointwise convergence of a sequence of increasing functions towards a continous function, then from the second Dini Theorem this convergence is uniform on any compact time interval. This shows that

$$
\sup _{0 \leq t \leq T \bar{C}}\left|\frac{1}{\mathbf{N}} P_{j}(\mathbf{N} t)-t\right| \longrightarrow 0 \text { a.s., as } \mathbf{N} \rightarrow \infty
$$

and the Proposition is established.

In what follows, $\|u\|$ denotes the norm of an $\ell$-dimensional vector u defined as follow $\|u\|:=\sum_{j=1}^{\ell}\left|u_{j}\right|$. Now we can prove the main result of this section. This law of large numbers is in fact a particular case of the general result in Britton \& Pardoux (2019). But since the proof is rather short, we prefered to include it for the convenience of the reader.

\section{Theorem 2.1 (Law of Large Numbers)}

Let $Z_{\mathbf{N}, \varepsilon}$ denote the solution of the $S D E$ (1.14) and $Z_{\varepsilon}$ the solution of the $O D E \frac{d Z_{\varepsilon}(t)}{d t}=b_{\varepsilon}\left(t, Z_{\varepsilon}(t)\right)$. Let us fix an arbitrary $T>0$ and assume that $\left\|Z_{\mathbf{N}, \varepsilon}(0)-Z_{\varepsilon}(0)\right\| \longrightarrow 0$, as $\mathbf{N} \rightarrow+\infty$.

Then $\sup _{0 \leq t \leq T}\left\|Z_{\mathbf{N}, \varepsilon}(t)-Z_{\varepsilon}(t)\right\| \longrightarrow 0$ a.s., as $\mathbf{N} \rightarrow+\infty$.

Proof : Let us define $M_{\mathbf{N}, \varepsilon}(t)=\sum_{j=1}^{k_{\varepsilon}} h_{j} M_{\mathbf{N}, \varepsilon}^{j}(t), t \in[0, T]$. We first note that

$$
\sup _{0 \leq t \leq T}\left\|M_{\mathbf{N}, \varepsilon}(t)\right\| \leq \sum_{j=1}^{k_{\varepsilon}}\left\|h_{j}\right\| \sup _{0 \leq t \leq T}\left|M_{\mathbf{N}, \varepsilon}^{j}(t)\right| .
$$

Hence from Proposition 2.1, we deduce that $\sup _{0 \leq t \leq T}\left\|M_{\mathbf{N}, \varepsilon}(t)\right\| \stackrel{\text { a.s }}{\longrightarrow} 0$, as $\mathbf{N} \rightarrow+\infty$. 
Next for any $r \in[0, T]$ we have

$$
\begin{aligned}
& \left\|b_{\varepsilon}\left(r, Z_{\mathbf{N}, \varepsilon}(r)\right)-b_{\varepsilon}\left(r, Z_{\varepsilon}(r)\right)\right\| \\
& \quad=2 \sum_{i=1}^{\ell} \beta\left(x_{i}\right)\left|\frac{S_{\mathbf{N}, \varepsilon}\left(r, x_{i}\right) I_{\mathbf{N}, \varepsilon}\left(r, x_{i}\right)}{S_{\mathbf{N}, \varepsilon}\left(r, x_{i}\right)+I_{\mathbf{N}, \varepsilon}\left(r, x_{i}\right)+R_{\mathbf{N}, \varepsilon}\left(r, x_{i}\right)}-\frac{S_{\varepsilon}\left(r, x_{i}\right) I_{\varepsilon}\left(r, x_{i}\right)}{S_{\varepsilon}\left(r, x_{i}\right)+I_{\varepsilon}\left(r, x_{i}\right)+R_{\varepsilon}\left(r, x_{i}\right)}\right| \\
& \quad+2 \sum_{i=1}^{\ell} \alpha\left(x_{i}\right)\left|I_{\mathbf{N}, \varepsilon}\left(r, x_{i}\right)-I_{\varepsilon}\left(r, x_{i}\right)\right|+\mu_{S} \sum_{i=1}^{\ell}\left|\Delta_{\varepsilon}\left(S_{\mathbf{N}, \varepsilon}\left(r, x_{i}\right)-S_{\varepsilon}\left(r, x_{i}\right)\right)\right| \\
& \quad+\mu_{I} \sum_{i=1}^{\ell}\left|\Delta_{\varepsilon}\left(I_{\mathbf{N}, \varepsilon}\left(r, x_{i}\right)-I_{\varepsilon}\left(r, x_{i}\right)\right)\right|+\mu_{R} \sum_{i=1}^{\ell}\left|\Delta_{\varepsilon}\left(R_{\mathbf{N}, \varepsilon}\left(r, x_{i}\right)-R_{\varepsilon}\left(r, x_{i}\right)\right)\right| .
\end{aligned}
$$

Then, the fact that $\beta$ and $\alpha$ are bounded leads to

$$
\begin{aligned}
\| b_{\varepsilon}\left(r, Z_{\mathbf{N}, \varepsilon}(r)\right)-b_{\varepsilon}\left(r, Z_{\varepsilon}(r)\right)|| \\
\quad \leq 2 \bar{\beta} \sum_{i=1}^{\ell}\left\{2\left|S_{\mathbf{N}, \varepsilon}\left(r, x_{i}\right)-S_{\varepsilon}\left(r, x_{i}\right)\right|+2\left|I_{\mathbf{N}, \varepsilon}\left(r, x_{i}\right)-I_{\varepsilon}\left(r, x_{i}\right)\right|+\left|R_{\mathbf{N}, \varepsilon}\left(r, x_{i}\right)-R_{\mathcal{\varepsilon}}\left(r, x_{i}\right)\right|\right\} \\
\quad+2 \bar{\alpha} \sum_{i=1}^{\ell}\left|I_{\mathbf{N}, \varepsilon}\left(r, x_{i}\right)-I_{\mathcal{\varepsilon}}\left(r, x_{i}\right)\right|+4 \mu_{S} \varepsilon^{-2} \sum_{i=1}^{\ell}\left|S_{\mathbf{N}, \varepsilon}\left(r, x_{i}\right)-S_{\varepsilon}\left(r, x_{i}\right)\right| \\
\quad+4 \mu_{I} \varepsilon^{-2} \sum_{i=1}^{\ell}\left|I_{\mathbf{N}, \varepsilon}\left(r, x_{i}\right)-I_{\varepsilon}\left(r, x_{i}\right)\right|+4 \mu_{R} \varepsilon^{-2} \sum_{i=1}^{\ell}\left|R_{\mathbf{N}, \varepsilon}\left(r, x_{i}\right)-R_{\varepsilon}\left(r, x_{i}\right)\right| \\
\leq C(\bar{\alpha}, \bar{\beta}, \bar{\mu}, \varepsilon) \| Z_{\mathbf{N}, \varepsilon}(r)-Z_{\varepsilon}(r)||, \text { where } \bar{\mu}=\max \left\{\mu_{S}, \mu_{I}, \mu_{R}\right\} .
\end{aligned}
$$

Hence we have for all $t \in[0, T]$

$$
\begin{aligned}
\left\|Z_{\mathbf{N}, \varepsilon}(t)-Z_{\varepsilon}(t)\right\| & \leq\left\|Z_{\mathbf{N}, \varepsilon}(0)-Z_{\varepsilon}(0)\right\|+\int_{0}^{t}\left\|b_{\varepsilon}\left(r, Z_{\mathbf{N}, \varepsilon}(r)\right)-b_{\varepsilon}\left(r, Z_{\varepsilon}(r)\right)\right\| d r+\left\|M_{\mathbf{N}, \varepsilon}(t)\right\| \\
& \leq\left(\left\|Z_{\mathbf{N}, \varepsilon}(0)-Z_{\mathcal{\varepsilon}}(0)\right\|+\left\|M_{\mathbf{N}, \varepsilon}(t)\right\|\right)+C(\bar{\alpha}, \bar{\beta}, \bar{\mu}, \varepsilon) \int_{0}^{t}\left\|Z_{\mathbf{N}, \varepsilon}(r)-Z_{\mathcal{\varepsilon}}(r)\right\| d r,
\end{aligned}
$$

and it follows from Gronwall's Lemma that

$$
\sup _{0 \leq t \leq T}\left\|Z_{\mathbf{N}, \varepsilon}(t)-Z_{\varepsilon}(t)\right\| \leq\left(\left\|Z_{\mathbf{N}, \varepsilon}(0)-Z_{\varepsilon}(0)\right\|+\sup _{0 \leq t \leq T}\left\|M_{\mathbf{N}, \varepsilon}(t)\right\|\right) \exp (C(\bar{\alpha}, \bar{\beta}, \bar{\mu}, \varepsilon) T) .
$$

This concludes the proof of the theorem, since $\left\|Z_{\mathbf{N}, \varepsilon}(0)-Z_{\mathcal{\varepsilon}}(0)\right\|+\sup _{0 \leq t \leq T}\left\|M_{\mathbf{N}, \varepsilon}(t)\right\| \longrightarrow 0$, as $\mathbf{N} \rightarrow+\infty$.

We have just shown that the solution of the stochastic model (1.14) converges a.s. locally uniformly in $t$ to the solution of the deterministic model (1.1), as $\mathbf{N} \rightarrow \infty, \varepsilon$ being fixed. If we then let $\varepsilon \rightarrow 0$, we know from Theorem 1.1 that the discrete deterministic system converges in $L^{\infty}(D)$ towards the system of PDEs 
on $\mathrm{D}$

$$
\left\{\begin{array}{l}
\frac{\partial \mathbf{s}}{\partial t}(t, x)=-\frac{\beta(x) \mathbf{s}(t, x) \mathbf{i}(t, x)}{\mathbf{s}(t, x)+\mathbf{i}(t, x)+\mathbf{r}(t, x)}+\mu_{S} \Delta \mathbf{s}(t, x) \\
\frac{\partial \mathbf{i}}{\partial t}(t, x)=\frac{\beta(x) \mathbf{s}(t, x) \mathbf{i}(t, x)}{\mathbf{s}(t, x)+\mathbf{i}(t, x)+\mathbf{r}(t, x)}-\alpha(x) \mathbf{i}(t, x)+\mu_{I} \Delta \mathbf{i}(t, x) \\
\frac{\partial \mathbf{r}}{\partial t}(t, x)=\alpha(x) \mathbf{i}(t, x)+\mu_{S} \Delta \mathbf{r}(t, x), \quad(t, x) \in(0, T) \times D .
\end{array}\right.
$$

\section{Law of Large Numbers in the Supremum norm}

In this section we let both the population size go to infinity and the mesh size $\varepsilon$ of the grid go to zero. Under the weak condition $\frac{\mathbf{N}}{\log (1 / \varepsilon)} \longrightarrow \infty$, we obtain that the stochastic spatial model converges in probability to the corresponding deterministic one.

Let us define $\mathcal{S}_{\mathbf{N}, \varepsilon}(t, x)=\sum_{i=1}^{\varepsilon^{-d}} S_{\mathbf{N}, \varepsilon}\left(t, x_{i}\right) \mathbf{1}_{V_{i}}(x), \quad \mathcal{I}_{\mathbf{N}, \varepsilon}(t, x)=\sum_{i=1}^{\varepsilon^{-d}} I_{\mathbf{N}, \varepsilon}\left(t, x_{i}\right) \mathbf{1}_{V_{i}}(x)$, and $\mathcal{R}_{\mathbf{N}, \varepsilon}(t, x)=\sum_{i=1}^{\varepsilon^{-d}} R_{\mathbf{N}, \varepsilon}\left(t, x_{i}\right) \mathbf{1}_{V_{i}}(x),(t, x) \in[0, T] \times D . \quad\left(\mathcal{S}_{\mathbf{N}, \varepsilon}, \mathcal{I}_{\mathbf{N}, \varepsilon}, \mathcal{R}_{\mathbf{N}, \varepsilon}\right)$ is solution of the SDEs

$$
\left\{\begin{aligned}
& \mathcal{S}_{\mathbf{N}, \varepsilon}(t, x)= \mathcal{S}_{\mathbf{N}, \varepsilon}(0, x)+\mu_{S} \int_{0}^{t} \Delta_{\varepsilon} \mathcal{S}_{\mathbf{N}, \varepsilon}(r, x) d r-\int_{0}^{t} \frac{\beta_{\varepsilon}(x) \mathcal{S}_{\mathbf{N}, \varepsilon}(r, x) \mathcal{I}_{\mathbf{N}, \varepsilon}(r, x)}{\mathcal{S}_{\mathbf{N}, \varepsilon}(r, x)+\mathcal{I}_{\mathbf{N}, \varepsilon}(r, x)+\mathcal{R}_{\mathbf{N}, \varepsilon}(r, x)} d r \\
&+\mathcal{M}_{\mathbf{N}, \varepsilon}^{S}(t, x) \\
& \mathcal{I}_{\mathbf{N}, \varepsilon}(t, x)= \mathcal{I}_{\mathbf{N}, \varepsilon}(0, x)+\mu_{I} \int_{0}^{t} \Delta_{\varepsilon} \mathcal{I}_{\mathbf{N}, \varepsilon}(r, x) d r+\int_{0}^{t} \frac{\beta_{\varepsilon}(x) \mathcal{S}_{\mathbf{N}, \varepsilon}(r, x) \mathcal{I}_{\mathbf{N}, \varepsilon}(r, x)}{\mathcal{S}_{\mathbf{N}, \varepsilon}(r, x)+\mathcal{I}_{\mathbf{N}, \varepsilon}(r, x)+\mathcal{R}_{\mathbf{N}, \varepsilon}(r, x)} d r \\
&-\int_{0}^{t} \alpha_{\varepsilon}(x) \mathcal{I}_{\mathbf{N}, \varepsilon}(r, x) d r+\mathcal{M}_{\mathbf{N}, \varepsilon}^{I}(t, x) \\
& \mathcal{R}_{\mathbf{N}, \varepsilon}(t, x)= \mathcal{R}_{\mathbf{N}, \varepsilon}(0, x)+\mu_{R} \int_{0}^{t} \Delta_{\varepsilon} \mathcal{R}_{\mathbf{N}, \varepsilon}(r, x) d r+\int_{0}^{t} \alpha_{\varepsilon}(x) \mathcal{I}_{\mathbf{N}, \varepsilon}(r, x) d r+\mathcal{M}_{\mathbf{N}, \varepsilon}^{R}(t, x) \\
&(t, x) \in[0, T] \times D
\end{aligned}\right.
$$

where $\quad \mathcal{M}_{\mathbf{N}, \varepsilon}^{S}(t, x)=-\frac{1}{\mathbf{N}} \sum_{i=1}^{\varepsilon^{-d}} \mathbf{M}_{x_{i}}^{i n f}\left(\mathbf{N} \int_{0}^{t} \frac{\beta\left(x_{i}\right) S_{\mathbf{N}, \varepsilon}\left(r, x_{i}\right) I_{\mathbf{N}, \varepsilon}\left(r, x_{i}\right)}{S_{\mathbf{N}, \varepsilon}\left(r, x_{i}\right)+I_{\mathbf{N}, \varepsilon}\left(r, x_{i}\right)+R_{\mathbf{N}, \varepsilon}\left(r, x_{i}\right)} d r\right) \mathbf{1}_{V_{i}}(x)$

$$
\begin{aligned}
& -\frac{1}{\mathbf{N}} \sum_{i=1}^{\varepsilon^{-d}} \sum_{y_{i} \sim x_{i}} \mathbf{M}_{S, x_{i}, y_{i}}^{m i g}\left(\frac{\mu_{S} \mathbf{N}}{\varepsilon^{2}} \int_{0}^{t} S_{\mathbf{N}, \varepsilon}\left(r, x_{i}\right) d r\right) \mathbf{1}_{V_{i}}(x) \\
& +\frac{1}{\mathbf{N}} \sum_{i=1}^{\varepsilon^{-d}} \sum_{y_{i} \sim x_{i}} \mathbf{M}_{S, y_{i}, x_{i}}^{m i g}\left(\frac{\mu_{S} \mathbf{N}}{\varepsilon^{2}} \int_{0}^{t} S_{\mathbf{N}, \varepsilon}\left(r, y_{i}\right) d r\right) \mathbf{1}_{V_{i}}(x),
\end{aligned}
$$




$$
\begin{aligned}
\mathcal{M}_{\mathbf{N}, \varepsilon}^{I}(t, x)= & \frac{1}{\mathbf{N}} \sum_{i=1}^{\varepsilon^{-d}} \mathbf{M}_{x_{i}}^{\text {inf }}\left(\mathbf{N} \int_{0}^{t} \frac{\beta\left(x_{i}\right) S_{\mathbf{N}, \varepsilon}\left(r, x_{i}\right) I_{\mathbf{N}, \varepsilon}\left(r, x_{i}\right)}{S_{\mathbf{N}, \varepsilon}\left(r, x_{i}\right)+I_{\mathbf{N}, \varepsilon}\left(r, x_{i}\right)+R_{\mathbf{N}, \varepsilon}\left(r, x_{i}\right)} d r\right) \mathbf{1}_{V_{i}}(x) \\
- & \frac{1}{\mathbf{N}} \sum_{i=1}^{\varepsilon^{-d}} \mathbf{M}_{x_{i}}^{r e c}\left(\mathbf{N} \int_{0}^{t} \alpha\left(x_{i}\right) I_{\mathbf{N}, \varepsilon}\left(r, x_{i}\right) d r\right) \mathbf{1}_{V_{i}}(x) \\
- & \frac{1}{\mathbf{N}} \sum_{i=1}^{\varepsilon^{-d}} \sum_{y_{i} \sim x_{i}} \mathbf{M}_{I, x_{i}, y_{i}}^{m i g}\left(\frac{\mu_{I} \mathbf{N}}{\varepsilon^{2}} \int_{0}^{t} I_{\mathbf{N}, \varepsilon}\left(r, x_{i}\right) d r\right) \mathbf{1}_{V_{i}}(x) \\
+ & \frac{1}{\mathbf{N}} \sum_{i=1}^{\varepsilon^{-d}} \sum_{y_{i} \sim x_{i}} \mathbf{M}_{I, y_{i}, x_{i}}^{m i g}\left(\frac{\mu_{I} \mathbf{N}}{\varepsilon^{2}} \int_{0}^{t} S_{\mathbf{N}, \varepsilon}\left(r, y_{i}\right) d r\right) \mathbf{1}_{V_{i}}(x) \\
\mathcal{M}_{\mathbf{N}, \varepsilon}^{R}(t, x) & =\frac{1}{\mathbf{N}} \sum_{i=1}^{\varepsilon^{-d}} \mathbf{M}_{x_{i}}^{r e c}\left(\mathbf{N} \int_{0}^{t} \alpha\left(x_{i}\right) I_{\mathbf{N}, \varepsilon}\left(r, x_{i}\right) d r\right) \mathbf{1}_{V_{i}}(x) \\
& -\frac{1}{\mathbf{N}} \sum_{i=1}^{\varepsilon^{-d}} \sum_{y_{i} \sim x_{i}} \mathbf{M}_{R, x_{i}, y_{i}}^{m i g}\left(\frac{\mu_{R} \mathbf{N}}{\varepsilon^{2}} \int_{0}^{t} R_{\mathbf{N}, \varepsilon}\left(r, x_{i}\right) d r\right) \mathbf{1}_{V_{i}}(x) \\
+ & \frac{1}{\mathbf{N}} \sum_{i=1}^{\varepsilon^{-d}} \sum_{y_{i} \sim x_{i}} \mathbf{M}_{R, y_{i}, x_{i}}^{m i g}\left(\frac{\mu_{R} \mathbf{N}}{\varepsilon^{2}} \int_{0}^{t} R_{\mathbf{N}, \varepsilon}\left(r, y_{i}\right) d r\right) \mathbf{1}_{V_{i}}(x)
\end{aligned}
$$

Here we set $\quad X_{\mathbf{N}, \varepsilon}=\left(\begin{array}{c}\mathcal{S}_{\mathbf{N}, \varepsilon} \\ \mathcal{I}_{\mathbf{N}, \varepsilon} \\ \mathcal{R}_{\mathbf{N}, \varepsilon}\end{array}\right)$ and $\mathcal{M}_{\mathbf{N}, \varepsilon}=\left(\begin{array}{c}\mathcal{M}_{\mathbf{N}, \varepsilon}^{S} \\ \mathcal{M}_{\mathbf{N}, \varepsilon}^{I} \\ \mathcal{M}_{\mathbf{N}, \varepsilon}^{R}\end{array}\right)$. Recall that $\mathcal{X}_{\varepsilon}=\left(\begin{array}{c}\mathcal{S}_{\varepsilon} \\ \mathcal{I}_{\varepsilon} \\ \mathcal{R}_{\varepsilon}\end{array}\right)$ and $X=$ $\left(\begin{array}{l}\mathbf{s} \\ \mathbf{i} \\ \mathbf{r}\end{array}\right)$

The main goal of this section is to prove the following result.

\section{Theorem 3.1 (Law of Large Numbers in Sup-norm)}

Let us assume that $(\varepsilon, \mathbf{N}) \rightarrow(0, \infty)$, in such way that
(i) $\frac{\mathbf{N}}{\log (1 / \varepsilon)} \longrightarrow \infty$ as $\mathbf{N} \rightarrow \infty$ and $\varepsilon \rightarrow 0$;
(ii) $\left\|X_{\mathbf{N}, \varepsilon}(0)-X(0)\right\|_{\infty} \longrightarrow 0$ in probability.

Then for all $T>0, \sup _{t \in[0, T]}\left\|X_{\mathbf{N}, \varepsilon}(t)-X(t)\right\|_{\infty} \longrightarrow 0$ in probability .

We prove the Theorem in the case $d=2$, but the result holds true in dimensions $\mathrm{d}=1,3$ as well, as we will explain below.

Since $\sup _{t \in[0, T]}\left\|\mathcal{X}_{\varepsilon}(t)-X(t)\right\|_{\infty} \longrightarrow 0$ by Theorem 1.1, clearly our Theorem will follow from

Proposition 3.1 For all $T>0$, $\sup _{t \in[0, T]}\left\|X_{\mathbf{N}, \varepsilon}(t)-\mathcal{X}_{\varepsilon}(t)\right\|_{\infty} \longrightarrow 0$ in probability, as $\mathbf{N} \rightarrow \infty$ and $\varepsilon \rightarrow 0$, in 
such a way that $\frac{\mathbf{N}}{\log (1 / \varepsilon)} \longrightarrow \infty$.

Proof : For all $t \in[0, T]$, We have

$$
\begin{gathered}
X_{\mathbf{N}, \varepsilon}(t)=X_{\mathbf{N}, \varepsilon}(0)+\int_{0}^{t} \widetilde{\Delta}_{\varepsilon} X_{\mathbf{N}, \varepsilon}(r) d r+\int_{0}^{t} G\left(X_{\mathbf{N}, \varepsilon}(r)\right) d r+\mathcal{M}_{\mathbf{N}, \varepsilon}(t), \\
\mathcal{X}_{\varepsilon}(t)=\mathcal{X}_{\varepsilon}(0)+\int_{0}^{t} \widetilde{\Delta}_{\varepsilon} \mathcal{X}_{\varepsilon}(r) d r+\int_{0}^{t} G\left(\mathcal{X}_{\varepsilon}(r)\right) d r, \\
X_{\mathbf{N}, \varepsilon}(t)-\mathcal{X}_{\varepsilon}(t)=\widetilde{\mathrm{T}}_{\varepsilon}(t)\left[X_{\mathbf{N}, \varepsilon}(0)-\mathcal{X}_{\varepsilon}(0)\right]+\int_{0}^{t} \widetilde{\mathrm{T}}_{\varepsilon}(t-r)\left[G\left(X_{\mathbf{N}, \varepsilon}(r)\right)-G\left(\mathcal{X}_{\varepsilon}(r)\right)\right] d r+Y_{\mathbf{N}, \varepsilon}(t), \\
\text { where again } \widetilde{\mathrm{T}}_{\varepsilon}(t) X_{\mathbf{N}, \varepsilon}=\left(\begin{array}{c}
\mathrm{T}_{\varepsilon, S}(t) \mathcal{S}_{\mathbf{N}, \varepsilon} \\
\mathrm{T}_{\varepsilon, I}(t) \mathcal{I}_{\mathbf{N}, \varepsilon} \\
\mathrm{T}_{\varepsilon, R}(t) \mathcal{R}_{\mathbf{N}, \varepsilon}
\end{array}\right) \text { and similarly for } \widetilde{\mathrm{T}}_{\varepsilon}(t) \mathcal{X}_{\varepsilon}, \cdots ; Y_{\mathbf{N}, \varepsilon}(t)=\left(\begin{array}{c}
Y_{\mathbf{N}, \varepsilon}^{S}(t) \\
Y_{\mathbf{N}, \varepsilon}^{I}(t) \\
Y_{\mathbf{N}, \varepsilon}^{R}(t)
\end{array}\right) \text { and } \\
Y_{\mathbf{N}, \varepsilon}^{S}(t)=\int_{0}^{t} \mathrm{~T}_{\varepsilon, S}(t-r) d \mathcal{M}_{\mathbf{N}, \varepsilon}^{S}(r), Y_{\mathbf{N}, \varepsilon}^{I}(t)=\int_{0}^{t} \mathrm{~T}_{\varepsilon, I}(t-r) d \mathcal{M}_{\mathbf{N}, \varepsilon}^{I}(r), Y_{\mathbf{N}, \varepsilon}^{R}(t)=\int_{0}^{t} \mathrm{~T}_{\varepsilon, R}(t-r) d \mathcal{M}_{\mathbf{N}, \varepsilon}^{R}(r) .
\end{gathered}
$$

As in the proof of Theorem 2.1, one can show that there is a constant $C(\bar{\beta}, \bar{\alpha})$ such that for all $r \in[0, T]$, we have

$$
\left\|G\left(X_{\mathbf{N}, \varepsilon}(r)\right)-G\left(\mathcal{X}_{\varepsilon}(r)\right)\right\|_{\infty} \leq C(\bar{\alpha}, \bar{\beta})\left\|X_{\mathbf{N}, \varepsilon}(r)-\mathcal{X}_{\varepsilon}(r)\right\|_{\infty}
$$

since G is globally Lipschitz. Using (3.2) and the fact that $\widetilde{\mathrm{T}}_{\varepsilon}$ is a contraction semigroup in $\left(L^{\infty}(D)\right)^{3}$, we have

$$
\left\|X_{\mathbf{N}, \varepsilon}(t)-\mathcal{X}_{\varepsilon}(t)\right\|_{\infty} \leq\left\|X_{\mathbf{N}, \varepsilon}(0)-\mathcal{X}_{\varepsilon}(0)\right\|_{\infty}+C(\bar{\alpha}, \bar{\beta}) \int_{0}^{t}\left\|X_{\mathbf{N}, \varepsilon}(r)-\mathcal{X}_{\varepsilon}(r)\right\|_{\infty} d r+\left\|Y_{\mathbf{N}, \varepsilon}(t)\right\|_{\infty} .
$$

It then follows from Gronwall's Lemma that

$$
\text { (3.3) } \sup _{t \in[0, T]}\left\|X_{\mathbf{N}, \varepsilon}(t)-\mathcal{X}_{\varepsilon}(t)\right\|_{\infty} \leq\left(\left\|X_{\mathbf{N}, \varepsilon}(0)-\mathcal{X}_{\varepsilon}(0)\right\|_{\infty}+\sup _{t \in[0, T]}\left\|Y_{\mathbf{N}, \varepsilon}(t)\right\|_{\infty}\right) e^{C(\bar{\alpha}, \bar{\beta}) T}
$$

Since $\left\|X_{\mathbf{N}, \varepsilon}(0)-\mathcal{X}_{\varepsilon}(0)\right\|_{\infty} \longrightarrow 0$ in probability, the Proposition follows from (3.3) and Proposition 3.2 below.

Proposition 3.2 For all $T>0$

(3.4) $\sup _{t \in[0, T]}\left\|Y_{\mathbf{N}, \varepsilon}(t)\right\|_{\infty} \longrightarrow 0$ in probability, as $\mathbf{N} \rightarrow \infty$ and $\varepsilon \rightarrow 0$, provided $\frac{\mathbf{N}}{\log (1 / \varepsilon)} \longrightarrow \infty$.

Before proving this Proposition, we first establish some technical Lemmas. 
Lemma 3.1 Let $f=\varepsilon^{-2} \mathbf{1}_{V_{i}}$. Then, for any $J \in\{S, I, R\}$

$$
\left\langle\left(\nabla_{\varepsilon}^{1,+} \mathrm{T}_{\varepsilon, J}(t) f\right)^{2}+\left(\nabla_{\varepsilon}^{1,-} \mathrm{T}_{\varepsilon, J}(t) f\right)^{2}+\left(\nabla_{\varepsilon}^{2,+} \mathrm{T}_{\varepsilon, J}(t) f\right)^{2}+\left(\nabla_{\varepsilon}^{2,-} \mathrm{T}_{\varepsilon, J}(t) f\right)^{2}+\left(\mathrm{T}_{\varepsilon, J}(t) f\right)^{2}, 1\right\rangle \leq h_{\varepsilon}(t)
$$

where

$$
\int_{0}^{t} h_{\varepsilon}(r) d r \leq C \varepsilon^{-2}+t
$$

Proof : For $f=\varepsilon^{-2} \mathbf{1}_{V_{i}}$ and $J \in\{S, I, R\}$, we have

$$
\begin{aligned}
\left\langle\left(\nabla_{\varepsilon}^{1,+} \mathrm{T}_{\varepsilon, J}(t) f\right)^{2}+\left(\nabla_{\varepsilon}^{2,+} \mathrm{T}_{\varepsilon, J}(t) f\right)^{2}, 1\right\rangle & =\left\langle\nabla_{\varepsilon}^{1,+} \mathrm{T}_{\varepsilon, J}(t) f, \nabla_{\varepsilon}^{1,+} \mathrm{T}_{\varepsilon, J}(t) f\right\rangle+\left\langle\nabla_{\varepsilon}^{2,+} \mathrm{T}_{\varepsilon, J}(t) f, \nabla_{\varepsilon}^{2,+} \mathrm{T}_{\varepsilon, J}(t) f\right\rangle \\
& =-\left\langle\nabla_{\varepsilon}^{1,-} \nabla_{\varepsilon}^{1,+} \mathrm{T}_{\varepsilon, J}(t) f, \mathrm{~T}_{\varepsilon, J}(t) f\right\rangle-\left\langle\nabla_{\varepsilon}^{2,-} \nabla_{\varepsilon}^{2,+} \mathrm{T}_{\varepsilon, J}(t) f, \mathrm{~T}_{\varepsilon, J}(t) f\right\rangle \\
& =-\left\langle\Delta_{\varepsilon} \mathrm{T}_{\varepsilon, J}(t) f, \mathrm{~T}_{\varepsilon, J}(t)(t) f\right\rangle .
\end{aligned}
$$

Using the facts that $\Delta_{\varepsilon} \mathrm{T}_{\varepsilon, J}(t) f=\mathrm{T}_{\varepsilon, J}(t) \Delta_{\varepsilon} f, \mathrm{~T}_{\varepsilon, J}(t)$ is self-adjoint and (1.3), we obtain

$$
\begin{aligned}
\left\langle\left(\nabla_{\varepsilon}^{1,+} \mathrm{T}_{\varepsilon, J}(t) f\right)^{2}+\left(\nabla_{\varepsilon}^{2,+} \mathrm{T}_{\varepsilon, J}(t) f\right)^{2}, 1\right\rangle & =-\left\langle\mathrm{T}_{\varepsilon, J}(t) \Delta_{\varepsilon} f, \mathrm{~T}_{\varepsilon, J}(t) f\right\rangle \\
& =-\left\langle\Delta_{\varepsilon} f, \mathrm{~T}_{\varepsilon, J}(2 t) f\right\rangle \\
& =\sum_{m}\left\langle f, \mathbf{f}_{m}^{\varepsilon}\right\rangle^{2} e^{-2 \lambda_{m, J}^{\varepsilon}} \lambda_{m, J}^{\varepsilon} \\
& \leq 4 \sum_{m} e^{-2 \lambda_{m, J}^{\varepsilon}} \lambda_{m, J}^{\varepsilon} .
\end{aligned}
$$

Similarly $\left\langle\left(\nabla_{\varepsilon}^{1,-} \mathrm{T}_{\varepsilon, J}(t) f\right)^{2}+\left(\nabla_{\varepsilon}^{2,-} \mathrm{T}_{\varepsilon, J}(t) f\right)^{2}, 1\right\rangle \leq 4 \sum_{m} e^{-2 \lambda_{m, J}^{\varepsilon} t} \lambda_{m, J}^{\varepsilon}$. Moreover, we have

$$
\begin{aligned}
\left\langle\left(\mathrm{T}_{\varepsilon, J}(t) f\right)^{2}, 1\right\rangle & =\left\langle\mathrm{T}_{\varepsilon, J}(2 t) f, f\right\rangle \\
& =1+\sum_{m \neq(0,0)} e^{-2 \lambda_{m, J}^{\varepsilon} t}\left\langle f, \mathbf{f}_{m}^{\varepsilon}\right\rangle^{2} \\
& \leq 1+4 \sum_{m \neq(0,0)} e^{-2 \lambda_{m, J}^{\varepsilon} t} .
\end{aligned}
$$

So, the result holds with $h_{\varepsilon}(t)=1+8 \sum_{m \neq(0,0)} e^{-2 \lambda_{m, J}^{\varepsilon} t}\left(\lambda_{m, J}^{\varepsilon}+1\right)$. 


$$
\begin{aligned}
\int_{0}^{t} h_{\varepsilon}(r) d r & \leq t+8 \sum_{m \neq(0,0)} \int_{0}^{+\infty} e^{-2 \lambda_{m, J}^{\varepsilon} r}\left(1+\lambda_{m, J}^{\varepsilon}\right) d r \\
& =t+4 \sum_{m \neq(0,0)}\left(1+\frac{1}{\lambda_{m, J}^{\varepsilon}}\right) \\
& \leq t+4 \sum_{\substack{1 \leq m_{1} \leq \varepsilon^{-1} \\
1 \leq m_{2} \leq \varepsilon^{-1}}}\left(1+\frac{1}{c\left(m_{1}^{2}+m_{2}^{2}\right)}\right) \\
& \leq t+4 \varepsilon^{-2}+\sum_{\substack{1 \leq m_{1} \leq \varepsilon^{-1} \\
1 \leq m_{2} \leq \varepsilon^{-1}}} \frac{2}{c} \\
& =t+\varepsilon^{-2}\left(4+\frac{2}{c}\right) .
\end{aligned}
$$

For any càdlàg process $Z$, let $\delta Z(t)=Z(t)-Z\left(t^{-}\right)$denote its jump at time $t$.

We shall need below the

Lemma 3.2 Let $M_{t}$ be a bounded martingale of finite variation defined on $\left[t_{0}, t_{1}\right]$ with $M_{t_{0}}=0$ and satisfying

(i) $M$ is right-continuous with left limits,

(ii) $\left|\delta M_{t}\right| \leq c$ for $t_{0} \leq t \leq t_{1}$, where $c$ is a positive constant,

(iii) $\sum_{t_{0} \leq s \leq t}\left(\delta M_{s}\right)^{2}-\int_{t_{0}}^{t} h(s) d s$ is a supermartingale, where $h$ is a positive deterministic function.

Then $\mathbb{E}\left(\exp \left(M_{t_{1}}\right)\right) \leq \exp \left(\frac{e^{c}}{2} \int_{t_{0}}^{t_{1}} h(s) d s\right)$.

\section{Proof :}

Let $f(x)=e^{x}$. We have $0 \leq f^{\prime \prime}(x+y)=f(x) f(y) \leq e^{c} f(x)$ for all $y \leq c$.

For $t_{0} \leq t \leq t_{1}$

$$
\begin{aligned}
f\left(M_{t}\right) & =1+\int_{t_{0}}^{t} f^{\prime}\left(M_{s^{-}}\right) d M_{s}+\sum_{t_{0} \leq s \leq t}\left(f\left(M_{s}\right)-f\left(M_{s^{-}}\right)-f^{\prime}\left(M_{s^{-}}\right) \delta M_{s}\right) \\
& \leq 1+\int_{t_{0}}^{t} f^{\prime}\left(M_{s^{-}}\right) d M_{s}+\frac{e^{c}}{2} \sum_{t_{0} \leq s \leq t} f\left(M_{s^{-}}\right)\left(\delta M_{s}\right)^{2} \\
& =1+\int_{t_{0}}^{t} f^{\prime}\left(M_{s^{-}}\right) d M_{s}+\frac{e^{c}}{2}\left(\sum_{t_{0} \leq s \leq t} f\left(M_{s^{-}}\right)\left(\delta M_{s}\right)^{2}-\int_{t_{0}}^{t} f\left(M_{s}\right) h(s) d s\right)+\frac{e^{c}}{2} \int_{t_{0}}^{t} f\left(M_{s}\right) h(s) d s
\end{aligned}
$$

where we used Taylor's formula and (ii) for the inequality.

From (iii) and the fact that $\int_{t_{0}}^{t} f^{\prime}\left(M_{s^{-}}\right) d M_{s}$ has mean zero, we deduce 


$$
\mathbb{E}\left(f\left(M_{t}\right)\right) \leq 1+\frac{e^{c}}{2} \int_{t_{0}}^{t} \mathbb{E}\left(f\left(M_{s}\right)\right) h(s) d s
$$

The result now follows from Gronwall's inequality.

Lemma 3.3 For any site $x_{i} \in D_{\varepsilon}$, the following are $\mathcal{F}_{t}^{\mathbf{N}, \varepsilon}$ mean zero martingales:

$$
\begin{aligned}
& \sum_{0 \leq r \leq t}\left(\delta S_{\mathbf{N}, \varepsilon}\left(r, x_{i}\right)\right)^{2}-\frac{1}{\mathbf{N}} \int_{0}^{t} \frac{\beta\left(x_{i}\right) S_{\mathbf{N}, \varepsilon}\left(r, x_{i}\right) I_{\mathbf{N}, \varepsilon}\left(r, x_{i}\right)}{S_{\mathbf{N}, \varepsilon}\left(r, x_{i}\right)+I_{\mathbf{N}, \varepsilon}\left(r, x_{i}\right)+R_{\mathbf{N}, \varepsilon}\left(r, x_{i}\right)} d r \\
& -\frac{4 \mu_{S}}{\mathbf{N} \varepsilon^{2}} \int_{0}^{t} S_{\mathbf{N}, \varepsilon}\left(r, x_{i}\right) d r-\frac{\mu_{S}}{\mathbf{N} \varepsilon^{2}} \int_{0}^{t}\left(\sum_{j=1}^{2} S_{\mathbf{N}, \varepsilon}\left(r, x_{i}+\varepsilon e_{j}\right)+\sum_{j=1}^{2} S_{\mathbf{N}, \varepsilon}\left(r, x_{i}-\varepsilon e_{j}\right)\right) d r
\end{aligned}
$$

$$
\begin{gathered}
\sum_{0 \leq r \leq t}\left(\delta I_{\mathbf{N}, \varepsilon}\left(r, x_{i}\right)\right)^{2}-\frac{1}{\mathbf{N}} \int_{0}^{t} \frac{\beta\left(x_{i}\right) S_{\mathbf{N}, \varepsilon}\left(r, x_{i}\right) I_{\mathbf{N}, \varepsilon}\left(r, x_{i}\right)}{S_{\mathbf{N}, \varepsilon}\left(r, x_{i}\right)+I_{\mathbf{N}, \varepsilon}\left(r, x_{i}\right)+R_{\mathbf{N}, \varepsilon}\left(r, x_{i}\right)} d r-\frac{1}{\mathbf{N}} \int_{0}^{t} \alpha\left(x_{i}\right) I_{\mathbf{N}, \varepsilon}\left(r, x_{i}\right) d r \\
-\frac{4 \mu_{I}}{\mathbf{N} \varepsilon^{2}} \int_{0}^{t} I_{\mathbf{N}, \varepsilon}\left(r, x_{i}\right) d r-\frac{\mu_{I}}{\mathbf{N} \varepsilon^{2}} \int_{0}^{t}\left(\sum_{j=1}^{2} I_{\mathbf{N}, \varepsilon}\left(r, x_{i}+\varepsilon e_{j}\right)+\sum_{j=1}^{2} I_{\mathbf{N}, \varepsilon}\left(r, x_{i}-\varepsilon e_{j}\right)\right) d r
\end{gathered}
$$

$$
\begin{array}{r}
\sum_{0 \leq r \leq t}\left(\delta R_{\mathbf{N}, \varepsilon}\left(r, x_{i}\right)\right)^{2}-\frac{1}{\mathbf{N}} \int_{0}^{t} \alpha\left(x_{i}\right) I_{\mathbf{N}, \varepsilon}\left(r, x_{i}\right) d r-\frac{4 \mu_{R}}{\mathbf{N} \varepsilon^{2}} \int_{0}^{t} R_{\mathbf{N}, \varepsilon}\left(r, x_{i}\right) d r \\
-\frac{\mu_{R}}{\mathbf{N} \varepsilon^{2}} \int_{0}^{t}\left(\sum_{j=1}^{2} R_{\mathbf{N}, \varepsilon}\left(r, x_{i}+\varepsilon e_{j}\right)+\sum_{j=1}^{2} R_{\mathbf{N}, \varepsilon}\left(r, x_{i}-\varepsilon e_{j}\right)\right) d r
\end{array}
$$

$$
\sum_{0 \leq r \leq t}\left(\delta S_{\mathbf{N}, \varepsilon}\left(r, x_{i}\right)\right)\left(\delta S_{\mathbf{N}, \varepsilon}\left(r, x_{i} \pm \varepsilon e_{j}\right)\right)+\frac{\mu_{S}}{\mathbf{N} \varepsilon^{2}} \int_{0}^{t}\left(S_{\mathbf{N}, \varepsilon}\left(r, x_{i}\right)+S_{\mathbf{N}, \varepsilon}\left(r, x_{i} \pm \varepsilon e_{j}\right)\right) d r
$$

$$
\sum_{0 \leq r \leq t}\left(\delta I_{\mathbf{N}, \varepsilon}\left(r, x_{i}\right)\right)\left(\delta I_{\mathbf{N}, \varepsilon}\left(r, x_{i} \pm \varepsilon e_{j}\right)\right)+\frac{\mu_{I}}{\mathbf{N} \varepsilon^{2}} \int_{0}^{t}\left(I_{\mathbf{N}, \varepsilon}\left(r, x_{i}\right) d r+I_{\mathbf{N}, \varepsilon}\left(r, x_{i} \pm \varepsilon e_{j}\right)\right) d r
$$

(3.11) $\sum_{0 \leq r \leq t}\left(\delta R_{\mathbf{N}, \varepsilon}\left(r, x_{i}\right)\right)\left(\delta R_{\mathbf{N}, \varepsilon}\left(r, x_{i} \pm \varepsilon e_{j}\right)\right)+\frac{\mu_{R}}{\mathbf{N} \varepsilon^{2}} \int_{0}^{t}\left(R_{\mathbf{N}, \varepsilon}\left(r, x_{i}\right) d r+R_{\mathbf{N}, \varepsilon}\left(r, x_{i} \pm \varepsilon e_{j}\right)\right) d r$ $j=1,2$.

Proof : The proof of this Lemma is based on the computation of the jumps. For (3.6), we have

$$
\begin{aligned}
\sum_{0 \leq r \leq t}\left(\delta S_{\mathbf{N}, \varepsilon}\left(r, x_{i}\right)\right)^{2} & =\frac{1}{\mathbf{N}^{2}} \mathrm{P}_{x_{i}}^{i n f}\left(\mathbf{N} \int_{0}^{t} \frac{\beta\left(x_{i}\right) S_{\mathbf{N}, \varepsilon}\left(r, x_{i}\right) I_{\mathbf{N}, \varepsilon}\left(r, x_{i}\right)}{S_{\mathbf{N}, \varepsilon}\left(r, x_{i}\right)+I_{\mathbf{N}, \varepsilon}\left(r, x_{i}\right)+R_{\mathbf{N}, \varepsilon}\left(r, x_{i}\right)} d r\right) \\
& +\sum_{y_{i} \sim x_{i}} \frac{1}{\mathbf{N}^{2}} \mathrm{P}_{S, x_{i}, y_{i}}^{m i g}\left(\frac{\mu_{S} \mathbf{N}}{\varepsilon^{2}} \int_{0}^{t} S_{\mathbf{N}, \varepsilon}\left(r, x_{i}\right) d r\right) \\
& +\sum_{y_{i} \sim x_{i}} \frac{1}{\mathbf{N}^{2}} \mathrm{P}_{S, y_{i}, x_{i}}^{m i g}\left(\frac{\mu_{S} \mathbf{N}}{\varepsilon^{2}} \int_{0}^{t} S_{\mathbf{N}, \varepsilon}\left(r, y_{i}\right) d r\right) .
\end{aligned}
$$


By writing each Poisson process as $M(t)+t$, we then have

$$
\begin{gathered}
\sum_{0 \leq r \leq t}\left(\delta S_{\mathbf{N}, \varepsilon}\left(r, x_{i}\right)\right)^{2}-\frac{1}{\mathbf{N}} \int_{0}^{t} \frac{\beta\left(x_{i}\right) S_{\mathbf{N}, \varepsilon}\left(r, x_{i}\right) I_{\mathbf{N}, \varepsilon}\left(r, x_{i}\right)}{S_{\mathbf{N}, \varepsilon}\left(r, x_{i}\right)+I_{\mathbf{N}, \varepsilon}\left(r, x_{i}\right)+R_{\mathbf{N}, \varepsilon}\left(r, x_{i}\right)} d r \\
-\frac{4 \mu_{S}}{\mathbf{N} \varepsilon^{2}} \int_{0}^{t} S_{\mathbf{N}, \varepsilon}\left(r, x_{i}\right) d r-\frac{\mu_{S}}{\mathbf{N} \varepsilon^{2}} \int_{0}^{t}\left(\sum_{j=1}^{2} S_{\mathbf{N}, \varepsilon}\left(r, x_{i}+\varepsilon e_{j}\right)+\sum_{j=1}^{2} S_{\mathbf{N}, \varepsilon}\left(r, x_{i}-\varepsilon e_{j}\right)\right) d r \\
=\frac{1}{\mathbf{N}^{2}} \mathbf{M}_{x_{i}}^{i n f}\left(\mathbf{N} \int_{0}^{t} \frac{\beta\left(x_{i}\right) S_{\mathbf{N}, \varepsilon}\left(r, x_{i}\right) I_{\mathbf{N}, \varepsilon}\left(r, x_{i}\right)}{S_{\mathbf{N}, \varepsilon}\left(r, x_{i}\right)+I_{\mathbf{N}, \varepsilon}\left(r, x_{i}\right)+R_{\mathbf{N}, \varepsilon}\left(r, x_{i}\right)} d r\right)+\sum_{y_{i} \sim x_{i}} \frac{1}{\mathbf{N}^{2}} \mathbf{M}_{S, x_{i}, y_{i}}^{m i g}\left(\frac{\mu_{S} \mathbf{N}}{\varepsilon^{2}} \int_{0}^{t} S_{\mathbf{N}, \varepsilon}\left(r, x_{i}\right) d r\right) \\
+\sum_{y_{i} \sim x_{i}} \frac{1}{\mathbf{N}^{2}} \mathbf{M}_{S, y_{i}, x_{i}}^{m i g}\left(\frac{\mu_{S} \mathbf{N}}{\varepsilon^{2}} \int_{0}^{t} S_{\mathbf{N}, \varepsilon}\left(r, y_{i}\right) d r\right),
\end{gathered}
$$

which is a martingale. The other statements are proved similarly.

The following result is a consequence of the previous Lemma.

Lemma 3.4 Let $\varphi \in H^{\varepsilon}$. The following are mean zero martingales

$$
\begin{aligned}
& \sum_{0 \leq r \leq t}\left(\delta\left\langle\mathcal{M}_{\mathbf{N}, \varepsilon}^{S}(r), \varphi\right\rangle\right)^{2}-\frac{\varepsilon^{2}}{\mathbf{N}} \int_{0}^{t}\left\langle\frac{\beta_{\varepsilon}(.) \mathcal{S}_{\mathbf{N}, \varepsilon}(r) \mathcal{I}_{\mathbf{N}, \varepsilon}(r)}{\mathcal{S}_{\mathbf{N}, \varepsilon}(r)+\mathcal{I}_{\mathbf{N}, \varepsilon}(r)+\mathcal{R}_{\mathbf{N}, \varepsilon}(r)}, \varphi^{2}\right\rangle d r \\
& -\frac{\mu_{S} \varepsilon^{2}}{\mathbf{N}} \int_{0}^{t}\left\langle\mathcal{S}_{\mathbf{N}, \varepsilon}(r),\left(\nabla_{\varepsilon}^{1,+} \varphi\right)^{2}+\left(\nabla_{\varepsilon}^{1,-} \varphi\right)^{2}+\left(\nabla_{\varepsilon}^{2,+} \varphi\right)^{2}+\left(\nabla_{\varepsilon}^{2,-} \varphi\right)^{2}\right\rangle d r
\end{aligned}
$$

$$
\sum_{0 \leq r \leq t}\left(\delta\left\langle\mathcal{M}_{\mathbf{N}, \varepsilon}^{I}(r), \varphi\right\rangle\right)^{2}-\frac{\varepsilon^{2}}{\mathbf{N}} \int_{0}^{t}\left\langle\frac{\beta_{\varepsilon}(.) \mathcal{S}_{\mathbf{N}, \varepsilon}(r) \mathcal{I}_{\mathbf{N}, \varepsilon}(r)}{\mathcal{S}_{\mathbf{N}, \varepsilon}(r)+\mathcal{I}_{\mathbf{N}, \varepsilon}(r)+\mathcal{R}_{\mathbf{N}, \varepsilon}(r)}, \varphi^{2}\right\rangle d r
$$

$-\frac{\varepsilon^{2}}{\mathbf{N}} \int_{0}^{t}\left\langle\alpha_{\varepsilon}(.) \mathcal{I}_{\mathbf{N}, \varepsilon}(r), \varphi^{2}\right\rangle d r-\frac{\mu_{I} \varepsilon^{2}}{\mathbf{N}} \int_{0}^{t}\left\langle\mathcal{I}_{\mathbf{N}, \varepsilon}(r),\left(\nabla_{\varepsilon}^{1,+} \varphi\right)^{2}+\left(\nabla_{\varepsilon}^{1,-} \varphi\right)^{2}+\left(\nabla_{\varepsilon}^{2,+} \varphi\right)^{2}+\left(\nabla_{\varepsilon}^{2,-} \varphi\right)^{2}\right\rangle d r$

$$
\begin{aligned}
& \sum_{0 \leq r \leq t}\left(\delta\left\langle\mathcal{M}_{\mathbf{N}, \varepsilon}^{R}(r), \varphi\right\rangle\right)^{2}-\frac{\varepsilon^{2}}{\mathbf{N}} \int_{0}^{t}\left\langle\alpha_{\varepsilon}(.) \mathcal{I}_{\mathbf{N}, \varepsilon}(r), \varphi^{2}\right\rangle d r \\
& \quad-\frac{\mu_{R} \varepsilon^{2}}{\mathbf{N}} \int_{0}^{t}\left\langle\mathcal{R}_{\mathbf{N}, \varepsilon}(r),\left(\nabla_{\varepsilon}^{1,+} \varphi\right)^{2}+\left(\nabla_{\varepsilon}^{1,-} \varphi\right)^{2}+\left(\nabla_{\varepsilon}^{2,+} \varphi\right)^{2}+\left(\nabla_{\varepsilon}^{2,-} \varphi\right)^{2}\right\rangle d r
\end{aligned}
$$

Proof : We give the proof for (3.12), those of (3.13) and (3.14) are similar. For all $r \leq t$, we have 
$\delta\left\langle\mathcal{M}_{\mathbf{N}, \varepsilon}^{S}(r), \varphi\right\rangle=\varepsilon^{2} \sum_{i=1}^{\varepsilon^{-2}} \delta \mathcal{S}_{\mathbf{N}, \varepsilon}\left(r, x_{i}\right) \varphi\left(x_{i}\right)$. Since for $y_{i} \neq x_{i} \pm \varepsilon e_{j},\left(\delta \mathcal{S}_{\mathbf{N}, \varepsilon}\left(r, x_{i}\right)\right)\left(\delta \mathcal{S}_{\mathbf{N}, \varepsilon}\left(r, y_{i}\right)\right)=0$, so

$$
\begin{aligned}
\left(\delta\left\langle\mathcal{M}_{\mathbf{N}, \varepsilon}^{S}(r), \varphi\right\rangle\right)^{2} & =\varepsilon^{4} \sum_{i=1}^{\varepsilon^{-2}}\left(\delta \mathcal{S}_{\mathbf{N}, \varepsilon}\left(r, x_{i}\right)\right)^{2} \varphi^{2}\left(x_{i}\right) \\
& +2 \varepsilon^{4} \sum_{i=1}^{\varepsilon^{-2}}\left(\delta \mathcal{S}_{\mathbf{N}, \varepsilon}\left(r, x_{i}\right)\right)\left(\delta \mathcal{S}_{\mathbf{N}, \varepsilon}\left(r, x_{i}+\varepsilon e_{1}\right)\right) \varphi\left(x_{i}\right) \varphi\left(x_{i}+\varepsilon e_{1}\right) \\
& +2 \varepsilon^{4} \sum_{i=1}^{\varepsilon^{-2}}\left(\delta \mathcal{S}_{\mathbf{N}, \varepsilon}\left(r, x_{i}\right)\right)\left(\delta \mathcal{S}_{\mathbf{N}, \varepsilon}\left(r, x_{i}-\varepsilon e_{1}\right)\right) \varphi\left(x_{i}\right) \varphi\left(x_{i}-\varepsilon e_{1}\right) \\
& +2 \varepsilon^{4} \sum_{i=1}^{\varepsilon^{-2}}\left(\delta \mathcal{S}_{\mathbf{N}, \varepsilon}\left(r, x_{i}\right)\right)\left(\delta \mathcal{S}_{\mathbf{N}, \varepsilon}\left(r, x_{i}+\varepsilon e_{2}\right)\right) \varphi\left(x_{i}\right) \varphi\left(x_{i}+\varepsilon e_{2}\right) \\
& +2 \varepsilon^{4} \sum_{i=1}^{\varepsilon^{-2}}\left(\delta \mathcal{S}_{\mathbf{N}, \varepsilon}\left(r, x_{i}\right)\right)\left(\delta \mathcal{S}_{\mathbf{N}, \varepsilon}\left(r, x_{i}-\varepsilon e_{2}\right)\right) \varphi\left(x_{i}\right) \varphi\left(x_{i}-\varepsilon e_{2}\right) .
\end{aligned}
$$

Using successively (3.6) and (3.9) from the previous lemma, we obtain

$$
\sum_{0 \leq r \leq t}\left(\delta \mathcal{S}_{\mathbf{N}, \varepsilon}\left(r, x_{i}\right)\right)^{2} \varphi^{2}\left(x_{i}\right)=\frac{1}{\mathbf{N}} \int_{0}^{t} \frac{\beta\left(x_{i}\right) \mathcal{S}_{\mathbf{N}, \varepsilon}\left(r, x_{i}\right) \mathcal{I}_{\mathbf{N}, \varepsilon}\left(r, x_{i}\right)}{\mathcal{S}_{\mathbf{N}, \varepsilon}\left(r, x_{i}\right)+\mathcal{I}_{\mathbf{N}, \varepsilon}\left(r, x_{i}\right)+\mathcal{R}_{\mathbf{N}, \varepsilon}\left(r, x_{i}\right)} \varphi^{2}\left(x_{i}\right) d r
$$

$+\frac{4 \mu_{S}}{\mathbf{N} \varepsilon^{2}} \int_{0}^{t} \mathcal{S}_{\mathbf{N}, \varepsilon}\left(r, x_{i}\right) \varphi^{2}\left(x_{i}\right) d r+\frac{\mu_{S}}{\mathbf{N} \varepsilon^{2}} \int_{0}^{t}\left[\sum_{j=1}^{2} \mathcal{S}_{\mathbf{N}, \varepsilon}\left(r, x_{i}+\varepsilon e_{j}\right)+\sum_{j=1}^{2} \mathcal{S}_{\mathbf{N}, \varepsilon}\left(r, x_{i}-\varepsilon e_{j}\right)\right] \varphi^{2}\left(x_{i}\right) d r+$ Martingale

and

$$
\begin{aligned}
& \sum_{0 \leq r \leq t}\left(\delta \mathcal{S}_{\mathbf{N}, \varepsilon}\left(r, x_{i}\right)\right)\left(\delta \mathcal{S}_{\mathbf{N}, \varepsilon}\left(r, x_{i} \pm \varepsilon e_{j}\right)\right) \varphi\left(x_{i}\right) \varphi\left(x_{i} \pm \varepsilon e_{j}\right) \\
& \quad=-\frac{\mu_{S}}{\mathbf{N} \varepsilon^{2}} \int_{0}^{t}\left(\mathcal{S}_{\mathbf{N}, \varepsilon}\left(r, x_{i}\right)+\mathcal{S}_{\mathbf{N}, \varepsilon}\left(r, x_{i} \pm \varepsilon e_{j}\right)\right) \varphi\left(x_{i}\right) \varphi\left(x_{i} \pm \varepsilon e_{j}\right) d r+\text { Martingale }
\end{aligned}
$$

Combining (3.15), (3.16) and (3.17), we deduce that

$$
\begin{aligned}
\sum_{0 \leq r \leq t}\left(\delta\left\langle\mathcal{M}_{\mathbf{N}, \varepsilon}^{S}(r), \varphi\right\rangle\right)^{2} & =\frac{\varepsilon^{2}}{\mathbf{N}} \int_{0}^{t}\left\langle\frac{\beta_{\varepsilon}(.) \mathcal{S}_{\mathbf{N}, \varepsilon}(r) \mathcal{I}_{\mathbf{N}, \varepsilon}(r)}{\mathcal{S}_{\mathbf{N}, \varepsilon}(r)+\mathcal{I}_{\mathbf{N}, \varepsilon}(r)+\mathcal{R}_{\mathbf{N}, \varepsilon}(r)}, \varphi^{2}\right\rangle d r \\
& +\frac{\mu_{S}}{\mathbf{N}} \int_{0}^{t}\left\langle 4 \mathcal{S}_{\mathbf{N}, \varepsilon}(r)+\sum_{j=1}^{2} \mathcal{S}_{\mathbf{N}, \varepsilon}\left(r, .+\varepsilon e_{j}\right)+\sum_{j=1}^{2} \mathcal{S}_{\mathbf{N}, \varepsilon}\left(r, .-\varepsilon e_{j}\right), \varphi^{2}\right\rangle d r \\
& -\frac{2 \mu_{S}}{\mathbf{N}} \int_{0}^{t}\left\langle\mathcal{S}_{\mathbf{N}, \varepsilon}(r), \sum_{j=1}^{2} \varphi(.) \varphi\left(.+\varepsilon e_{j}\right)+\sum_{j=1}^{2} \varphi(.) \varphi\left(.-\varepsilon e_{j}\right)\right\rangle d r+\text { Martingale }
\end{aligned}
$$

which can also be written as 


$$
\begin{aligned}
\sum_{0 \leq r \leq t}\left(\delta\left\langle\mathcal{M}_{\mathbf{N}, \varepsilon}^{S}(r), \varphi\right\rangle\right)^{2}=\frac{\varepsilon^{2}}{\mathbf{N}} \int_{0}^{t}\left\langle\frac{\beta_{\varepsilon}(.) \mathcal{S}_{\mathbf{N}, \varepsilon}(r) \mathcal{I}_{\mathbf{N}, \varepsilon}(r)}{\mathcal{S}_{\mathbf{N}, \varepsilon}(r)+\mathcal{I}_{\mathbf{N}, \varepsilon}(r)+\mathcal{R}_{\mathbf{N}, \varepsilon}(r)}, \varphi^{2}\right\rangle d r \\
-\frac{\mu_{S} \varepsilon^{2}}{\mathbf{N}} \int_{0}^{t}\left\langle\mathcal{S}_{\mathbf{N}, \varepsilon}(r),\left(\nabla_{\varepsilon}^{1,+} \varphi\right)^{2}+\left(\nabla_{\varepsilon}^{1,-} \varphi\right)^{2}+\left(\nabla_{\varepsilon}^{2,+} \varphi\right)^{2}+\left(\nabla_{\varepsilon}^{2,-} \varphi\right)^{2}\right\rangle d r+\text { Martingale. }
\end{aligned}
$$

The following Lemma generalizes Lemma 3.4 in the case of a non constant $\varphi \in C\left(\mathbb{R}_{+} ; H^{\varepsilon}\right)$.

Lemma 3.5 The assertion of Lemma 3.4 is valid if $\varphi \in C\left(\mathbb{R}_{+} ; H^{\varepsilon}\right)$.

Proof : The general result follows by approximation. $\varphi$ being continuous with respect to $t$, there exists a sequence $\left(\varphi_{j}\right)_{1 \leq j \leq n}$ of step functions which converges to $\varphi$ locally uniformly in $[0, \infty)$. It then suffices to consider the case where $\varphi$ is a step function which we assume from now on. There exists a sequence $0=t_{0}<t_{1}<t_{2}<\cdots<t_{n}=t$ such that $\varphi\left(t, x_{i}\right)=\sum_{j=1}^{n} \varphi_{j}\left(x_{i}\right) \mathbf{1}_{\left(t_{j-1}, t_{j}\right]}(t)$, where $\varphi_{j} \in H^{\varepsilon}$, for all $j=1, \cdots, n$. Applying Lemma 3.4 on each interval $\left(t_{j-1}, t_{j}\right]$ and summing for all $j \in\{1, \cdots, n\}$ yields to the result.

Now we are in a position to give the

\section{Proof of Proposition 3.2}

Let us fix $\bar{t} \in(0, T], i \in\left\{1, \cdots, \varepsilon^{-2}\right\}$ and we use the notation $f=\varepsilon^{-2} \mathbf{1}_{V_{i}}$. We define $\bar{m}_{\mathbf{N}, \varepsilon}^{S}(t):=\left\langle\int_{0}^{t} \mathrm{~T}_{\varepsilon, S}(\bar{t}-r) d \mathcal{M}_{\mathbf{N}, \varepsilon}^{S}(r), f\right\rangle, \quad 0 \leq t \leq \bar{t}$. Note that the process $\left\{\bar{m}_{\mathbf{N}, \varepsilon}^{S}(t), t \in[0, \bar{t}]\right\}$ is a mean zero martingale and we have

$\bar{m}_{\mathbf{N}, \varepsilon}^{S}(\bar{t})=Y_{\mathbf{N}, \varepsilon}^{S}(\bar{t})$. We have $\sum_{0 \leq r \leq t}\left(\delta \bar{m}_{\mathbf{N}, \varepsilon}^{S}(r)\right)^{2}=\sum_{0 \leq r \leq t}\left(\left\langle\delta \mathcal{M}_{\mathbf{N}, \varepsilon}^{S}(r), \mathrm{T}_{\varepsilon, S}(\bar{t}-r) f\right\rangle\right)^{2}$. From Lemma 3.5, we have that

$$
\sum_{0 \leq r \leq t}\left(\delta \bar{m}_{\mathbf{N}, \varepsilon}^{S}(r)\right)^{2}-\int_{0}^{t} g_{\varepsilon}(r) d r
$$

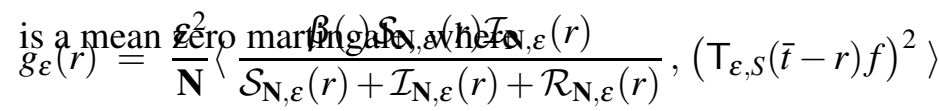

$$
\begin{aligned}
& +\frac{\mu_{S} \varepsilon^{2}}{\mathbf{N}}\left\langle\mathcal{S}_{\mathbf{N}, \varepsilon}(r),\left(\nabla_{\varepsilon}^{1,+} \mathrm{T}_{\varepsilon, S}(\bar{t}-r) f\right)^{2}+\left(\nabla_{\varepsilon}^{1,-} \mathrm{T}_{\varepsilon, S}(\bar{t}-r) f\right)^{2}+\left(\nabla_{\varepsilon}^{2,+} \mathrm{T}_{\varepsilon, S}(\bar{t}-r) f\right)^{2}+\left(\nabla_{\varepsilon}^{2,-} \mathrm{T}_{\varepsilon, S}(\bar{t}-r) f\right)^{2}\right\rangle .
\end{aligned}
$$

We have

$$
\begin{aligned}
& g_{\varepsilon}(r) \leq \frac{\bar{\beta} \varepsilon^{2}}{\mathbf{N}}\left\langle 1,\left(\mathrm{~T}_{\varepsilon, S}(\bar{t}-r) f\right)^{2}\right\rangle \\
& \quad+\frac{C \mu_{S} \varepsilon^{2}}{\mathbf{N}}\left\langle 1,\left(\nabla_{\mathcal{\varepsilon}}^{1,+} \mathrm{T}_{\mathcal{\varepsilon}, S}(\bar{t}-r) f\right)^{2}+\left(\nabla_{\mathcal{\varepsilon}}^{1,-} \mathrm{T}_{\mathcal{\varepsilon}, S}(\bar{t}-r) f\right)^{2}+\left(\nabla_{\mathcal{\varepsilon}}^{2,+} \mathrm{T}_{\varepsilon, S}(\bar{t}-r) f\right)^{2}+\left(\nabla_{\mathcal{\varepsilon}}^{2,-} \mathrm{T}_{\varepsilon, S}(\bar{t}-r) f\right)^{2}\right\rangle .
\end{aligned}
$$


For $\theta \in[0,1]$, we define $m_{\mathbf{N}, \varepsilon}^{S}(t)=\theta \mathbf{N} \bar{m}_{\mathbf{N}, \varepsilon}^{S}(t) . m_{\mathbf{N}, \varepsilon}^{S}$ is a mean zero martingale. Furthermore

$$
\begin{aligned}
\left|\delta m_{\mathbf{N}, \varepsilon}^{S}\right| & \leq \theta \mathbf{N}\left\|\mathrm{T}_{\varepsilon, S}(\bar{t}-t) \delta \mathcal{M}_{\mathbf{N}, \varepsilon}(t)\right\|_{\infty} \int_{D} f(x) d x \\
& \leq 1
\end{aligned}
$$

It follows from Lemma 3.1 and Lemma 3.2 that

$$
\mathbb{E}\left(\exp \left(m_{\mathbf{N}, \varepsilon}^{S}(\bar{t})\right)\right) \leq \exp \left[\frac{e}{2} \theta^{2} C\left(\bar{\beta}, \mu_{S}\right) \mathbf{N} \varepsilon^{2}\left(\bar{t}+C \varepsilon^{-2}\right)\right]
$$

It then follows that for any site $x_{i} \in D_{\varepsilon}, \eta>0$

$$
\begin{aligned}
\mathbb{P}\left(Y_{\mathbf{N}, \varepsilon}^{S}\left(\bar{t}, x_{i}\right)>\eta\right) & =\mathbb{P}\left(\theta \mathbf{N} Y_{\mathbf{N}, \varepsilon}^{S}\left(\bar{t}, x_{i}\right)>\theta \mathbf{N} \eta\right) \\
& \leq \mathbb{E}\left[\exp \left(\theta \mathbf{N} Y_{\mathbf{N}, \varepsilon}^{S}\left(\bar{t}, x_{i}\right)\right)\right] \exp (-\theta \mathbf{N} \eta) \\
& \leq \exp [\theta \mathbf{N}(C(T) \theta-\eta)] \quad \text { with } C(T)=\frac{e}{2} C\left(\bar{\beta}, \mu_{S}\right)(T+C)
\end{aligned}
$$

The optimal $\theta$ is $\theta=\frac{\eta}{2 C(T)}$, hence $\mathbb{P}\left(Y_{\mathbf{N}, \varepsilon}^{S}\left(\bar{t}, x_{i}\right)>\eta\right) \leq \exp \left(-a \eta^{2} \mathbf{N}\right)$, with $a=\frac{1}{4 C(T)}$. We can make a similar computation for $\mathbb{P}\left(-Y_{\mathbf{N}, \varepsilon}^{S}\left(\bar{t}, x_{i}\right)>\eta\right)$ to show that $\mathbb{P}\left(-Y_{\mathbf{N}, \varepsilon}^{S}\left(\bar{t}, x_{i}\right)>\eta\right) \leq \exp \left(-a \eta^{2} \mathbf{N}\right)$.

Hence for all $t \in[0, T]$ and $i \in\left\{1, \cdots, \varepsilon^{-2}\right\}$, we have

$$
\mathbb{P}\left(\left|Y_{\mathbf{N}, \varepsilon}^{S}\left(t, x_{i}\right)\right|>\eta\right) \leq 2 \exp \left(-a \eta^{2} \mathbf{N}\right)
$$

Since $\left\|Y_{\mathbf{N}, \varepsilon}^{S}(t)\right\|_{\infty}=\sup _{i}\left|Y_{\mathbf{N}, \varepsilon}^{S}\left(t, x_{i}\right)\right|$

$$
\begin{aligned}
\mathbb{P}\left(\left\|Y_{\mathbf{N}, \varepsilon}^{S}(t)\right\|_{\infty}>\eta\right) & \leq \sum_{i=1}^{\varepsilon^{-2}} \mathbb{P}\left(\left|Y_{\mathbf{N}, \varepsilon}^{S}\left(t, x_{i}\right)\right|>\eta\right) \\
& \leq 2 \varepsilon^{-2} \exp \left(-a \eta^{2} \mathbf{N}\right) .
\end{aligned}
$$

We now show that an inequality similar to (3.19) holds with $\left\|Y_{\mathbf{N}, \varepsilon}^{S}(t)\right\|_{\infty}$ replaced by $\sup _{t \in[0, T]}\left\|Y_{\mathbf{N}, \varepsilon}^{S}(t)\right\|_{\infty}$.

To this end, we divide $[0, T]$ into $\varepsilon^{-2}$ intervals $\left[n T \varepsilon^{2},(n+1) T \varepsilon^{2}\right], 0 \leq n \leq \varepsilon^{-2}-1$.

For $t \in\left[n T \varepsilon^{2},(n+1) T \varepsilon^{2}\right]$, we have

$Y_{\mathbf{N}, \varepsilon}^{S}(t)=Y_{\mathbf{N}, \varepsilon}^{S}\left(n T \varepsilon^{2}\right)+\int_{n T \varepsilon^{2}}^{t} \Delta_{\varepsilon} Y_{\mathbf{N}, \varepsilon}^{S}(r) d r+\tilde{m}_{\mathbf{N}, \varepsilon}^{S}(t)$, where $\tilde{m}_{\mathbf{N}, \varepsilon}^{S}(t)=\mathcal{M}_{\mathbf{N}, \varepsilon}^{S}(t)-\mathcal{M}_{\mathbf{N}, \varepsilon}^{S}\left(n T \varepsilon^{2}\right)$.

We have

$$
\left\|Y_{\mathbf{N}, \varepsilon}^{S}(t)\right\|_{\infty} \leq\left\|Y_{\mathbf{N}, \varepsilon}^{S}\left(n T \varepsilon^{2}\right)\right\|_{\infty}+8 \varepsilon^{-2} \int_{n T \varepsilon^{2}}^{t}\left\|Y_{\mathbf{N}, \varepsilon}^{S}(r)\right\|_{\infty} d r+\left\|\tilde{m}_{\mathbf{N}, \varepsilon}^{S}(t)\right\|_{\infty}
$$

so Gronwall's inequality implies that 
(3.21) $\sup _{t \in\left[n T \varepsilon^{2},(n+1) T \varepsilon^{2}\right]}\left\|Y_{\mathbf{N}, \varepsilon}^{S}(t)\right\|_{\infty} \leq\left(\left\|Y_{\mathbf{N}, \varepsilon}^{S}\left(n T \varepsilon^{2}\right)\right\|_{\infty}+\sup _{t \in\left[n T \varepsilon^{2},(n+1) T \varepsilon^{2}\right]}\left\|\tilde{m}_{\mathbf{N}, \varepsilon}^{S}(t)\right\|_{\infty}\right) \exp (8 T)$.

We now fix $i \in\left\{1, \cdots, \varepsilon^{-2}\right\}, \theta \in[0,1]$ and set $m_{\mathbf{N}, \varepsilon}^{S}(t)=\theta \mathbf{N} \tilde{m}_{\mathbf{N}, \varepsilon}^{S}(t)$. It follows from Lemma 3.3 that

$$
\begin{aligned}
& \sum_{n T \varepsilon^{2} \leq r \leq t}\left(\delta m_{\mathbf{N}, \varepsilon}^{S}(r)\right)^{2}-\frac{\mu_{S}}{\varepsilon^{2}} \theta^{2} \mathbf{N} \int_{n T \varepsilon^{2}}^{t}\left(\sum_{y \sim x_{i}} \mathcal{S}_{\mathbf{N}, \varepsilon}(r, y)+4 \mathcal{S}_{\mathbf{N}, \varepsilon}\left(r, x_{i}\right)\right) d r \\
& \quad-\theta^{2} \mathbf{N} \int_{n T \varepsilon^{2}}^{t} \frac{\beta\left(x_{i}\right) \mathcal{S}_{\mathbf{N}, \varepsilon}\left(r, x_{i}\right) \mathcal{I}_{\mathbf{N}, \varepsilon}\left(r, x_{i}\right)}{\mathcal{S}_{\mathbf{N}, \varepsilon}\left(r, x_{i}\right)+\mathcal{I}_{\mathbf{N}, \varepsilon}\left(r, x_{i}\right)+\mathcal{R}_{\mathbf{N}, \varepsilon}\left(r, x_{i}\right)} d r
\end{aligned}
$$

is a mean zero martingale and $\left|\delta m_{\mathbf{N}, \varepsilon}^{S}(t)\right| \leq 1$. Furthermore, for $n T \varepsilon^{2}<t \leq(n+1) T \varepsilon^{2}$

$$
\begin{array}{r}
\frac{\mu_{S}}{\varepsilon^{2}} \theta^{2} \mathbf{N} \int_{n T \varepsilon^{2}}^{t}\left(\sum_{y \sim x_{i}} \mathcal{S}_{\mathbf{N}, \varepsilon}(r, y)+4 \mathcal{S}_{\mathbf{N}, \varepsilon}\left(r, x_{i}\right)\right) d r+\mathbf{N} \theta^{2} \int_{n T \varepsilon^{2}}^{t} \frac{\beta\left(x_{i}\right) \mathcal{S}_{\mathbf{N}, \varepsilon}\left(r, x_{i}\right) \mathcal{I}_{\mathbf{N}, \varepsilon}\left(r, x_{i}\right)}{\mathcal{S}_{\mathbf{N}, \varepsilon}\left(r, x_{i}\right)+\mathcal{I}_{\mathbf{N}, \varepsilon}(r)+\mathcal{R}_{\mathbf{N}, \varepsilon}\left(r, x_{i}\right)} d r \\
\leq C\left(\bar{\beta}, \mu_{S}\right) T \mathbf{N} \theta^{2} .
\end{array}
$$

Hence by Lemma 3.2, it follows that $\mathbb{E}\left[\exp \left(m_{\mathbf{N}, \varepsilon}^{S}\left((n+1) T \varepsilon^{2}\right)\right)\right] \leq \exp \left[C\left(\bar{\beta}, \mu_{S}\right) \mathbf{N} \theta^{2} T\right]$.

It then follows from Doob's inequality that

$$
\begin{aligned}
\mathbb{P}\left(\sup _{t \in\left[n T \varepsilon^{2},(n+1) T \varepsilon^{2}\right]} \tilde{m}_{\mathbf{N}, \varepsilon}^{S}\left(t, x_{i}\right) \geq \eta\right) & \leq \mathbb{E}\left[\exp \left(m_{\mathbf{N}, \varepsilon}^{S}\left((n+1) T \varepsilon^{2}\right)\right)\right] \exp (-\theta \mathbf{N} \eta) \\
& \leq \exp [\theta \mathbf{N}(C(T) \theta-\eta)]
\end{aligned}
$$

Choosing $\theta=\frac{\eta}{2 C(T)}$, we deduce that

$$
\mathbb{P}\left(\sup _{t \in\left[n T \varepsilon^{2},(n+1) T \varepsilon^{2}\right]} \tilde{m}_{\mathbf{N}, \varepsilon}^{S}\left(t, x_{i}\right) \geq \eta\right) \leq \exp \left(-a \eta^{2} \mathbf{N}\right), \text { where } a=\frac{1}{4 C(T)} .
$$

The same hold for $-\tilde{m}_{\mathbf{N}, \varepsilon}^{S}\left(t, x_{i}\right)$. Consequently

$$
\mathbb{P}\left(\sup _{t \in\left[n T \varepsilon^{2},(n+1) T \varepsilon^{2}\right]}\left\|\tilde{m}_{\mathbf{N}, \varepsilon}^{S}\left(t, x_{i}\right)\right\|_{\infty} \geq \eta\right) \leq 2 \varepsilon^{-2} \exp \left(-a \eta^{2} \mathbf{N}\right) .
$$

Combining the inequalities (3.19), (3.21) and (3.23), we obtain

$$
\mathbb{P}\left(e^{-8 T} \sup _{t \in\left[n T \varepsilon^{2},(n+1) T \varepsilon^{2}\right]}\left\|Y_{\mathbf{N}, \varepsilon}^{S}(t)\right\|_{\infty} \geq \eta\right) \leq 4 \varepsilon^{-2} \exp \left(-a \frac{\eta^{2}}{4} \mathbf{N}\right)
$$


from which we deduce that

$$
\begin{aligned}
\mathbb{P}\left(e^{-8 T} \sup _{t \in[0, T]}\left\|Y_{\mathbf{N}, \varepsilon}^{S}(t)\right\|_{\infty} \geq \eta\right) & \leq \sum_{n=0}^{\varepsilon^{-2}-1} \mathbb{P}\left(e^{-8 T} \sup _{t \in\left[n T \varepsilon^{2},(n+1) T \varepsilon^{2}\right]}\left\|Y_{\mathbf{N}, \varepsilon}^{S}(t)\right\|_{\infty} \geq \eta\right) \\
& \leq 4 \varepsilon^{-4} \exp \left(-a \frac{\eta^{2}}{4} \mathbf{N}\right) .
\end{aligned}
$$

Since $\frac{\mathbf{N}}{\log (1 / \varepsilon)} \longrightarrow+\infty$ implies that $\varepsilon^{-4} \exp \left(-a \eta^{2} \mathbf{N}\right) \longrightarrow 0$, we have proved that $\sup _{t \in[0, T]}\left\|Y_{\mathbf{N}, \varepsilon}^{S}(t)\right\|_{\infty} \longrightarrow 0$ in probability. The same arguments show that $\sup _{t \in[0, T]}\left\|Y_{\mathbf{N}, \varepsilon}^{I}(t)\right\|_{\infty}+\sup _{t \in[0, T]}\left\|Y_{\mathbf{N}, \varepsilon}^{R}(t)\right\|_{\infty} \longrightarrow 0$ in probability as $\mathbf{N} \rightarrow \infty$ and $\varepsilon \rightarrow 0$, under our standing assumption. Finally, we have shown that $\sup _{t \in[0, T]}\left\|Y_{\mathbf{N}, \varepsilon}(t)\right\|_{\infty} \longrightarrow 0$ in probability, which completes the proof of the Proposition.

\section{Remark 1}

The law of large numbers in sup-norm remains true in dimensions $d=1,3$. To see that, it suffices to remark that $\Delta_{\varepsilon}=\sum_{j=1}^{d} \nabla_{\varepsilon}^{j,-} \nabla_{\varepsilon}^{j,+}$ has always $\varepsilon^{-d}$ bounded eigenvectors. In this case the Lemma 3.1 become

$$
\left\langle\sum_{j=1}^{d}\left(\nabla_{\varepsilon}^{j,+} \mathrm{T}_{\varepsilon, J}(t) f\right)^{2}+\sum_{j=1}^{d}\left(\nabla_{\varepsilon}^{j,-} \mathrm{T}_{\varepsilon, J}(t) f\right)^{2}+\left(\mathrm{T}_{\varepsilon, J}(t) f\right)^{2}, 1\right\rangle \leq h_{\varepsilon}(t)
$$

where $\int_{0}^{t} h_{\varepsilon}(r) d r \leq C \varepsilon^{-d}+t$. Hence (3.25) becomes $\mathbb{P}\left(e^{-8 T} \sup _{t \in[0, T]}\left\|Y_{\mathbf{N}, \varepsilon}^{S}(t)\right\|_{\infty} \geq \eta\right) \leq 4 \varepsilon^{-d-2} \exp \left(-a \frac{\eta^{2}}{4} \mathbf{N}\right)$.

Moreover, the result holds for periodic boundary conditions. Indeed, in this case, the eigenvectors of the Laplace operator are the product of the one-dimensional eigenvectors

$$
\begin{gathered}
\varphi_{n}(x)=\left\{\begin{array}{r}
1, \text { for } n=0, \\
\sqrt{2} \cos (n \pi x), \text { for } n>0 \text { and even }
\end{array}\right. \\
\psi_{n}(x)=\sqrt{2} \sin (n \pi x), \text { for } n>0 \text { and even. }
\end{gathered}
$$

\section{Remark 2}

We conclude that, by two laws of large numbers, the consistency of the various models has been established.

In a furture work, we will study the fluctuations of the stochastic model around its deterministic law of large numbers limit. 
Acknowledgments. The authors are deeply indebted to the referee for a careful reading and several suggestions that greatly improved the paper.

\section{References}

[1] F. B. Agusto (2017a), Mathematical model of Ebola transmission dynamics with relapse and reinfection, Math. Biosci., 287, 48-59.

[2] F. B. Agusto, S. Bewick and W. F. Fagan (2017b), Mathematical model for Zika virus dynamics with sexual transmission route, Ecological Complexity, 29 , 61-81.

[3] L. J. S. Allen, F. Brauer, P. Van den Driessche, \& J. Wu (2008). Mathematical epidemiology (Vol. 1945). Berlin: Springer.

[4] L. J. S. Allen, B. M. Bolker, Y. Lou \& A. L. Nevai (2008). Asymptotic profiles of the steady states for an SIS epidemic reaction-diffusion model. Discrete and Continuous Dynamical Systems, 21, No. $1,1-20$.

[5] L. J. S. Allen, B. M. Bolker, Y. Lou \& A. L. Nevai (2007). Asymptotic profiles of the steady states for an SIS epidemic patch model. SIAM Journal on Applied Mathematics, 67, No. 5, 1283-1309.

[6] H. Andersson and T. Britton (2000). Stochastic epidemic models and their statistical analysis. Springer Lecture Notes in Statistics. New York: Springer Verlag

[7] L. Arnold and M. Theodosopulu (1980). Deterministic limit of the stochastic model of chemical reactions with diffusion. Adv. Appl. Prob., 12, No. 2, 367-379.

[8] D. Blount (1992), Law of large numbers in the supremum norm for a chemical reaction with diffusion, The Annals of Applied Probability, 2, No. 1, 131-141

[9] T. Britton and E. Pardoux (2019). Stochastic epidemic in a homogeneous community, arxiv:1808.05350, to appear.

[10] M. J. Debussche, A., \& M. J. N. Nankep (2017). A Law of Large Numbers in the Supremum Norm for a Multiscale Stochastic Spatial Gene Network. arXiv preprint arXiv:1711.06010.

[11] Z. Du and R. Peng (2016), A priori $L^{\infty}$ estimates for solutions of a class of reaction-diffusion systems, J. Math. Biol., 72 , 1429-1439

[12] T. Kato (1966). Perturbation Theory for Linear Operators. Springer-Verlag

[13] W.O. Kermack and A.G. McKendrick (1927), Proc. Roy. Soc. A 115 , 700. Reprinted in Bull. Math. Biol. 53 (1991) 33. 
[14] P. Kotelenez (1986). Gaussian approximation to the nonlinear reaction-diffusion equation. Report 146, Universität Bremen Forschungsschwerpunkt Dynamische Systemes.

[15] H. L. Smith (1995). Monotone dynamical systems: an introduction to the theory of competitive and cooperative systems (No. 41). American Mathematical Soc..

[16] K. Yamazaki (2018a), Threshold dynamics of reaction-diffusion partial differential equations model of Ebola virus disease, Int. J. Biomath., 11, 1850108.

[17] K. Yamazaki (2018b), Global well-posedness of infectious disease models without life-timme immunity: the cases of cholera and avian influenza, Math. Med. Biol., 35 , 428-445

[18] K. Yamazaki and X. Wang (2016). Global well-posedness and asymptotic behavior of solutions to a reaction-convection-diffusion cholera epidemic model. Discrete Contin. Dyn. Syst. Ser. B, 21, 1297-1316. doi: 10.3934/dcdsb.2016.21.1297

[19] G. F. Webb (1981). A reaction-diffusion model for a deterministic epidemic, Journal of Mathematical Analysis and Applications 84, 150-161 